\title{
On the application of dynamic zone flamelet model to large eddy simulation of supersonic hydrogen flame
}

\author{
Wei Yao ${ }^{a, b}$ \\ ${ }^{a}$ Key Laboratory of High Temperature Gas Dynamics, Institute of Mechanics, CAS, No.15 Beisihuanxi Road, Beijing \\ 100190, China \\ ${ }^{\mathrm{b}}$ School of Engineering Science, University of Chinese Academy of Science, Beijing 100049, China
}

\section{H I G H L I G H T S}

- Decouple turbulence-chemistry interaction based on dynamic zone flamelet concept.

- Both zone division and flamelet evolve dynamically based on local conditions.

- Model accuracy and efficiency confirmed by the DNS validation.

\section{A R T I C L E I N F O}

Article history:

Received 9 March 2020

Received in revised form

1 May 2020

Accepted 20 May 2020

Available online 8 July 2020

Keywords:

Dynamic zone flamelet model

Supersonic hydrogen jet

Large eddy simulation

Supersonic combustion

Turbulent combustion model

\begin{abstract}
A B S T R A C T
A dynamic zone flamelet model (DZFM) is proposed to decouple the turbulence-chemistry interactions in supersonic combustion modeling based on local statistical homogeneity assumption. The whole turbulent combustion field is divided into a finite number of control zones, and the chemical status in each zone is represented by a local flamelet, which evolves according to the spatial exchange with its neighbors, chemical reactions controlled by representative temperature, and differential diffusion in the mixture fraction space. Both the zone division and its representative flamelet are dynamically updated for better representing the local chemical status. The zone-based flamelet model is then applied to large eddy simulation of a supersonic hydrogen flame based on 106.23 million cells and 30,0 $\times 91$ flamelet zones. The predictions agree well with the DNS calculation, with the auto-ignition process and the flame lift-off phenomenon well reproduced. Sensitivity and cost analysis under different numbers of flamelet zones were also conducted.
\end{abstract}

๑ 2020 Hydrogen Energy Publications LLC. Published by Elsevier Ltd. All rights reserved.

\section{Introduction}

Conventional flamelet model is generally considered inapplicable for supersonic combustion modeling, because of the complex turbulence-chemistry interaction at high Reynolds number typically above $5 \times 10^{5}$. In most hypersonic propulsion systems, the flow residence time in microseconds is short enough to be even comparable to the chemical time scales. The close in time scales implies strong turbulence-chemistry interaction (TCI) and intense scalar fluctuations. Under such circumstances, it is well known that the effect of the fluctuations of species concentration and temperature on the

E-mail address: weiyao@imech.ac.cn. 
nonlinear reaction rate cannot be neglected. Universal decoupling models, like the flamelet model (FM) [1], for the separately resolving of turbulence and chemistry in supersonic combustion, are thus not plausible to be applied. For this reason, finite-rate chemistry models with full species transport are usually used in supersonic combustion modeling, but the tremendously additional computational cost would then be required to solve the chemistry cell by cell as well as the species transport equations one by one.

By decoupling the chemistry solving with the flow solving, the flamelet model [1] allows the modeling of complex chemistry at the minimum additional cost compared with the frozen flow modeling. However, the applicability of the flamelet model generally assumes a thin reaction zone that is less disturbed by the turbulence and where the chemistry is close to equilibrium, so that the reactive scalar $\mathrm{Y}$ can be well related to the mixture fraction $\xi$ together with additional indicator variable(s) (like scalar dissipation rate $\chi$ ) as $Y=Y(\xi, \chi)$. The asymptotic relation may evolve in time $t$ as $Y=Y(\xi, \chi, t)$, if some adaptation time is needed to the variation of indicator variable(s), which is the concept of unsteady flamelet [2]. When the flame front thickness exceeds Kolmogorov length scale or the eddy turnover velocity $v^{\prime}$ (Taylor-scale velocity) exceeds the laminar flame speed $S_{l}$, the flame front is convoluted to form corrugated reaction zones, and may further be broken up into distributed isolated reaction zone "islands" [3]. Although asymptotic relation may be still valid for each fragmented flamelet (or thin reaction zone), due to the intersection and random orientation of those fragmented flamelets, no exact asymptotic relation can be derived for the ensemble of a flame region containing a large number of fragmented flamelets. To extend the applicability of the flamelet model, Representative Interactive Flamelets (RIF) model has been proposed [4,5] to correlate $Y$ and $\xi$ using the concept of representative flamelets. The applicability of RIF is not limited to thin reaction zones only, but is applicable to non-equilibrium, transient and heterogeneous reaction processes [6]. However, in the conventional FM, the RIF, and the latest developed multi-flamelet RIF [5], the flamelet(s) are all applied to the whole combustion field.

Due to the heterogeneous nature of supersonic combustion modelings, the instantaneous values of Y may not be well correlated with the conserved scalar $\xi$ for the whole combustion field. In supersonic combustion modeling, the complex chemistry interacts with flows covering a wide range of speeds from subsonic to supersonic. The flow speed inside the combustor usually has a Mach number below $3[7,8]$. However, hypersonic combustion under even higher Mach number is possible in some local regions inside the scramjet, for example, the combustion starts inside the high-Mach inlet when adopting inlet injection technique [9], and incomplete combustion may continue in the accelerating flow through the nozzle section with a high expansion ratio. In supersonic combustion, the chemistry is in highly nonequilibrium status under the short flow residence time, and the mixing is mostly incomplete for nonpremixed cases. Thus both the turbulence and combustion chemistry is extremely heterogeneous, and different turbulence-chemistry modes exist in different flow regions. Thereby, a single flamelet is not enough to accurately represent the correlation between $\mathrm{Y}$ and $\xi$ on the whole field, i.e., there is a statistical dependence of local chemical status on the physical coordinates other than mixture fraction. Under such circumstances, the ensemble correlation between $Y$ and $\xi$ has been distorted at different levels by local turbulent stirring; conventional flamelet based models are theoretically inapplicable while finite-rate models are usually used instead.

Instead, using the concept of local flamelets for different flow regions, where the chemical status is influenced by different turbulence-chemistry interaction modes, can be a more suitable solution, as long as the local statistical homogeneity can be ensured through a proper zone division $[10,11]$. The highly nonequilibrium chemistry, the scalar dissipation, and the differential diffusion are the three main causes for spatial variations of chemical status versus mixture fraction. The turbulent motion exerts influence on the flamelet evolution solely through adjusting the scalar dissipation rate. As mentioned before, if the flamelet adapts instantaneously to the variation of scalar dissipation rate (or strain rate), the pretabulated steady flamelet model can be used [2]. However, such convenience cannot be enjoyed in the modeling of supersonic reacting jet involving strong strain rates, where the characteristic diffusion time scale is comparable to the largest chemical time scale. In highly transient supersonic combustion, the local statistical homogeneity also varies from time to time. Such statistical inhomogeneity in supersonic combustion has been evidenced by the temporal $[12,13]$ and spatial [14-19] variation of reacting scalar profiles in the mixture fraction space. In this study, flamelet modeling based on dynamic zone division is developed to allow a better local statistical homogeneity and to diminish the redistribution effect of conditional variances. The model is then applied to model a supersonic hydrogen jet flame for preliminary validation.

\section{Formulation of dynamic zone flamelet model (DZFM)}

Defining a local flamelet within each zone, the conditional species mass fraction can be calculated as $\mathrm{Q}_{i}=\left\langle\mathrm{Y}_{i}\right| \xi(x, t)=\eta$, $x \in$ zone $\rangle$, where $\eta$ is the sampling variable in mixture fraction space, $x$ represents the physical coordinate,,$x \in$ zone implying that the conditional average is confined within the zone. Correspondingly, the instantaneous mass fraction is related to $Q_{i}$ as

$Y_{i}(x, t)=Q_{i}(\eta=\xi(x, t), x \in$ zone,$t)+Y_{i}^{\prime}(x \in$ zone,$t)$

Here, $Y_{i}^{\prime}$ represents the deviation of instantaneous value from the conditional average within the current zone. Certainly, it has $\left\langle Q_{i}^{\prime}\right| \eta, x \in$ zone $\rangle=0$, and the zone-averaged $\left\langle\mathrm{Q}_{i}^{\prime}\right\rangle_{\text {zone }}=\int\left\langle\mathrm{Q}_{i}^{\prime}\right| \eta, x \in$ zone $\rangle \mathrm{P}(\eta) d \eta=0$, with $\mathrm{P}(\eta)$ the probability density function (PDF) describing the distribution of instantaneous $\xi$ within the zone. Note that as the zone shrinks, the fluctuation never disappears but occurs at a low level since the local statistical homogeneity would be more valid.

For completeness, the instantaneous equations for mixture fraction $\xi$ and the species mass fraction $Y_{i}$ with different diffusivities are listed below, 
$\rho \frac{\partial \xi}{\partial t}+\rho \vec{U} \cdot \nabla \xi=\nabla \cdot\left(\rho D_{\xi} \nabla \xi\right)$

$\rho \frac{\partial Y_{i}}{\partial t}+\rho \vec{U} \cdot \nabla Y_{i}=\nabla \cdot\left(\rho D_{i} \nabla Y_{i}\right)+\rho W_{i}$

with $\rho$ is the density, $\vec{U}$ is the velocity vector, $D_{\xi}$ and $D_{i}$ represents the diffusivities of the mixture parcel and individual species respectively, and $W_{i}$ denotes the reaction rate with unit $s^{-1}$.

Substitute the differentiation of Eq. (1) into the instantaneous governing equation for species $Y_{i}(E q(2))$ and using the conservation law of passive mixture fraction $\xi$ (Eq. (3)), it arrives that,

$$
\begin{aligned}
& \rho \frac{\partial Q_{i}}{\partial t}+\rho \vec{U} \cdot \nabla Q_{i}-\rho D_{i}(\nabla \xi)^{2} \frac{\partial^{2} Q_{i}}{\partial \eta^{2}}+\frac{\partial Q_{i}}{\partial \eta} \\
& \left(\rho \frac{\partial \xi}{\partial t}+\rho \vec{U} \cdot \nabla \xi-\nabla \cdot\left(\rho D_{\xi} \nabla \xi\right)\right)+\left(1-\frac{D_{i}}{D_{\xi}}\right) \nabla \cdot\left(\rho D_{\xi} \nabla \xi\right) \frac{\partial Q_{i}}{\partial \eta} \\
& +\left(\rho \frac{\partial Q_{i}^{\prime}}{\partial t}+\rho \vec{U} \cdot \nabla Q_{i}^{\prime}-\nabla \cdot\left(\rho D_{i} \nabla Q_{i}^{\prime}\right)\right)-\rho D_{i} \nabla \xi \cdot \nabla\left(\frac{\partial Q_{i}}{\partial \eta}\right) \\
& -\rho D_{i} \nabla^{2} Q_{i}=\rho W_{i}
\end{aligned}
$$

Taking the average of Eq. (4) on conditions that 1) $\xi(x, t)=\eta$ and 2) within the zone $x \in$ zone, it yields the final governing equation for $Q_{i}$ as,

$$
\begin{aligned}
& \rho_{\eta} \frac{\partial Q_{i}}{\partial t}+\langle\rho \vec{U} \mid \eta\rangle_{\text {zone }} \cdot \nabla Q_{i}+E_{Z F M}=\rho_{\eta} \frac{D_{i}}{D_{\xi}}\langle\chi \mid \eta\rangle_{\text {zone }} \frac{\partial^{2} Q_{i}}{\partial \eta^{2}}+\rho_{\eta}\left(\frac{D_{i}}{D_{\xi}}-1\right) M_{\eta} \frac{\partial Q_{i}}{\partial \eta} \\
& +\rho_{\eta}\left\langle W_{i} \mid \eta\right\rangle
\end{aligned}
$$

$$
\begin{aligned}
& \text { with } E_{\text {ZFM }}=\langle\underbrace{\left\langle\partial Q_{i}^{\prime} / \partial t+\vec{U} \cdot \nabla Q_{i}^{\prime}-D \nabla^{2} Q_{i}^{\prime}\right.}_{\text {ex }_{Y}} \mid \eta\rangle_{\text {zone }}-\left\langle\rho D \nabla \xi \cdot \nabla\left(\frac{\partial Q_{i}}{\partial \eta}\right) \mid \eta\right\rangle_{\text {zone }} \\
& -\left\langle\nabla \cdot\left(\rho D \nabla Q_{i}\right) \mid \eta\right\rangle_{\text {zone }}
\end{aligned}
$$

and $\chi$ is the scalar dissipation rate defined as $\chi=D_{\xi}(\nabla \xi)^{2}, \rho_{\eta}=$ $\langle\rho \mid \eta\rangle$, and the conditional diffusion $\mathrm{M}_{\eta}=\left\langle\nabla \cdot\left(\rho D_{\xi} \nabla \xi\right) \mid \eta\right\rangle_{\text {zone }}$. The second term on the LHS in Eq. (5) Represents the convective transport of conditional variables or local flamelet in a flowing manner among neighbor zones, such that the flamelets in the downstream inherit the chemical status of the preceding flamelet from the upstream, which is the physical picture in simulating the ignition process and flame lift-off phenomenon. While in the multi-flamelet RIF [5], the ignition is realized by reinitializing a new flamelet as mixed-but-unburnt but inheriting the preceding flamelet temperature. Eq. (5) describes the evolution of local flamelet within a zone due to convection in the physical space, differential diffusion in the mixture fraction space, and chemical reactions in the species space. The conventional flamelet can be considered as a special zone flamelet model taking the whole computational domain as a single zone. The last two terms in Eq. (6) correspond to the macro-transport by molecular diffusion. Following the similar analysis in the derivation of Conditional Moment Closure (CMC) [20], $\left\langle\rho D_{i} \nabla \xi \cdot \nabla\left(\partial Q_{i} / \partial \eta\right) \mid \eta\right\rangle_{\text {zone }} \sim \rho D_{i} \xi D_{i}^{-1 / 2} \cdot Q_{i} \sim D_{i}^{1 / 2} \sim R e^{-1 / 2}, \quad$ and $\left\langle\nabla \cdot\left(\rho D \nabla Q_{i}\right) \mid \eta\right\rangle_{\text {zone }} \sim \rho D \cdot Q_{i} \sim R e^{-1}$ thus can both be neglected in supersonic flows typically with a large Re $>10^{5}$. Even in some local subsonic flow regions [7,8,21] embedded in the supersonic flow, the lower bound of $\operatorname{Re}>1000[3,22,23]$ can still approximately valid the assumption. In supersonic flows, the velocity and density are largely influenced by the Mach number in addition to the mixture fraction, thus the approximations of $\langle\vec{U} \mid \eta\rangle=\vec{U}$ and $\langle\rho \mid \eta\rangle=\rho$ are used. Then, it arrives at that,

$$
\begin{aligned}
\int\left\langle e_{Y} \mid \eta\right\rangle_{\text {zone }} P(\eta) d \eta=\left\langle e_{Y}\right\rangle_{\text {zone }}= & \rho \partial\left\langle Q_{i}^{\prime}\right\rangle_{\text {zone }} / \partial t+\rho \vec{U} \cdot \nabla\left\langle Q_{i}^{\prime}\right\rangle_{\text {zone }} \\
& -\nabla \cdot\left\langle\rho D \nabla Q_{i}^{\prime}\right\rangle_{\text {zone }} \\
= & 0
\end{aligned}
$$

In the application, the zone division is dynamically updated with the mixture fraction field to make each zone corresponding to a narrow mixture fraction bin $\eta \in\left[\bar{\xi}_{\text {zone }}-\Delta \xi / 2\right.$, $\bar{\xi}_{\text {zone }}+\Delta \xi / 2$ ], where $\bar{\xi}_{\text {zone }}$ is the zone averaged mixture fraction, and $\Delta \xi$ represents the variation range of the instantaneous mixture fraction within a zone. Through refining the zone division, $\Delta \xi$ shrinks and the PDF distribution within each zone collapses to a Dirac delta function centered on $\eta=\bar{\xi}_{\text {zone }}$, i.e., $\int\left\langle e_{Y} \mid \eta\right\rangle_{\text {zone }} \delta\left(\eta=\bar{\xi}_{\text {zone }}\right) d \eta=\left\langle e_{Y} \mid \eta=\bar{\xi}_{\text {zone }}\right\rangle \approx\left\langle e_{Y} \mid \eta=\xi\right\rangle_{\text {zone }}=0$. It implies that the conditional variance terms in Eq. (6) can be neglected through a dynamic zone division adaptive to the mixture fraction field. Previous numerical validation has confirmed the shrinking of $\Delta \xi$ and the collapse of PDF from a $\beta$ function to a Dirac delta function.

The implementation details of DZFM are briefly introduced here. The conditional scalar dissipation rate $\langle\chi \mid \eta\rangle$ is modeled by amplitude mapping closure (AMC) model [24] as in the generic CMC model, the mean scalar dissipation rate $\tilde{\chi}$ is calculated by its relationship with the mixture fraction variance $\tilde{\chi}=C_{D} \widetilde{\xi^{\prime \prime 2}} / \tau_{\Delta}$ [25] with $C_{D}=4.0, \widetilde{\xi^{\prime \prime 2}}$ the mixture fraction variance and $\tau_{\Delta}$ the subgrid time scale. The conditional diffusion $\mathrm{M}_{\eta}$ is related to $\langle\chi \mid \eta\rangle$ [26]. A historical statistics approach [10] similar to the one used in Ref. [27] is developed to estimate the flamelet temperature, which significantly saves the computational cost of solving the conditional energy equation with numerous unclosed conditional sub-models. Those zone-statistical flamelet temperature controls the reaction progress within each own zone, implying that a reacting mixture parcel, whose status is described by the current zone flamelet, will experience reactions under different intensities when transporting from one zone to the other zones from a lagrangian viewpoint. Contrary to the standard flamelet model, the flame temperature was iteratively computed from the total enthalpy based on that $\tilde{H}=$ $\sum \tilde{Y}_{\alpha} H_{\alpha}(\tilde{T})$, where $\tilde{H}$ and $H_{\alpha}$ are the absolute enthalpy for the mixture and each individual species under the mass fraction of $\tilde{Y}_{\alpha}$, rather than retrieving directly from the integration of the flamelet table. In such way [28-32], various compressibility and heat-loss effects, including isoentropic compression/expansion, shock wave, viscous dissipation, and wall heat transfer etc., can be accounted for in solving the flow governing equation of $\tilde{H}$, based on the simple fact that the temperature in supersonic flow is not only correlated with the 
mixture fraction but also significantly influenced by the variation of Mach number, the entropy loss, and other nonadiabatic effects. The local compressibility effects on the chemical reactions have been accounted for by passing the zoneaveraged pressure and the zone-sampled temperature from the flow module, which can better represent the local perturbations due to compressibility than the rescaling approach [32]. The compressibility corrections on the chemical reaction rate [3] and the subgrid-scale (SGS) viscosity [33] were found to have negligible effects for Mach number below 2 [34], although may need to be activated for higher Mach numbers. The integration from conditional species stored in the flamelet to the unconditional values is conducted based on $\beta$-function PDF, which is a function of the mean mixture fraction $\tilde{\xi}$ and its variance $\widetilde{\xi^{\prime \prime}}$. Favre mean equations for $\tilde{\xi}$ and $\widetilde{\xi^{\prime \prime 2}}$ are respectively solved from their governing equations [35].

\section{Numerical implementation}

\section{Governing equations}

The unsteady and three-dimensional Favre-averaged compressible reactive Navier-Stokes equations (rNSE) are solved for a set of conservative variables $\left(\bar{\rho}, \tilde{u}_{i}, \tilde{H}_{t}, \tilde{Y}_{\alpha}\right)[36,37]$,

$\frac{\partial \bar{\rho}}{\partial t}+\frac{\partial \bar{\rho} \tilde{u}_{j}}{\partial x_{j}}=0$

$\frac{\partial \bar{\rho} \tilde{u}_{i}}{\partial t}+\frac{\partial \bar{\rho} \tilde{u}_{j} \tilde{u}_{i}}{\partial x_{j}}+\frac{\partial \bar{p}}{\partial x_{i}}-\frac{\partial \tilde{\tau}_{i j}}{\partial x_{j}}=-\frac{\partial \tau_{i j}}{\partial x_{j}}$

$\frac{\partial \bar{\rho} \tilde{H}_{t}}{\partial t}+\frac{\partial \bar{\rho} \tilde{u}_{j} \tilde{H}_{t}}{\partial x_{j}}-\frac{\partial}{\partial x_{j}}\left(\bar{\rho} D_{T} \frac{\partial \tilde{H}_{t}}{\partial x_{j}}+\sum_{\alpha=1}^{L} \bar{\rho}_{\alpha} \frac{\partial \tilde{Y}_{\alpha}}{\partial x_{j}} \tilde{H}_{\alpha}\right)-\frac{\partial \bar{p}}{\partial t}-\frac{\partial \tilde{u}_{j} \tilde{\tau}_{i j}}{\partial x_{j}}=$

$-\frac{\partial \Psi_{T, j}}{\partial x_{j}}$

$\frac{\partial \bar{\rho} \tilde{Y}_{\alpha}}{\partial t}+\frac{\partial \bar{\rho} \tilde{u}_{j} \tilde{Y}_{\alpha}}{\partial x_{j}}-\frac{\partial}{\partial x_{j}}\left(\bar{\rho} D_{\alpha} \frac{\partial \tilde{Y}_{\alpha}}{\partial x_{j}}\right)=-\frac{\partial \Psi_{\alpha, j}}{\partial x_{j}}+\bar{\omega}_{\alpha}$

$\bar{p}=\bar{\rho} R \tilde{T}$

$\tilde{H}_{\mathrm{t}}=\tilde{\mathrm{H}}+\frac{1}{2} \tilde{u}_{\mathrm{i}} \tilde{u}_{i}=\tilde{H}^{0}+\int_{0}^{T} C_{p} d T+\frac{1}{2} \tilde{u}_{i} \tilde{u}_{i}$

Here the bar "-" and the tilde " " represent averaged and Favre-averaged quantities respectively, $t$ denotes the time, $x_{i}$ is the Cartesian coordinate in direction $i, \bar{\rho}$ is the density, $\tilde{u}_{i}$ is the velocity component in $x_{i}$ direction (spatial dimension $i=1$, $2,3), \bar{p}$ is the pressure, $\tilde{\tau}_{i j}$ is the viscous stress tensor, $\tilde{H}_{t}=\tilde{H}+$ $0.5 \tilde{u}_{i}^{2}$ is the total absolute enthalpy obtained as the sum of the absolute enthalpy $\tilde{H}$ and the resolved kinetic energy, the absolute enthalpy $\tilde{H}$ is calculated as the sum of the formation enthalpy $\tilde{H}^{0}$ at standard reference state and the sensible enthalpy change from the reference temperature to $T, \tilde{Y}_{\alpha}$ is the mass fraction of species $\alpha(\alpha=1, \ldots, L$, with $L$ the total species number), the specific heat $C_{p}$ is a function of species concentrations and temperature, $\bar{\omega}_{\alpha}$ is the averaged mass production rate of chemical species $\alpha$ in the unit of $\mathrm{kg} \cdot \mathrm{m}^{-3} \cdot \mathrm{s}^{-1}, \tilde{T}$ is the temperature, $D_{\alpha}$ is mixture-averaged mass diffusivity of species $\alpha$ calcualted based on the Fitered values $\left(\bar{p}, \tilde{T}, \tilde{Y}_{\alpha}\right), D_{T}$ is the thermal diffusivity calcualted based on the Fitered values, $R=R_{u} / W$ is the gas constant, $R_{u}=$ $8.314 \mathrm{~J} \cdot \mathrm{mol}^{-1} \cdot \mathrm{K}^{-1}$ is the universal gas constant, $\mathrm{W}=\left(\sum_{\alpha=1}^{L} \mathrm{Y}_{\alpha} / \mathrm{W}_{\alpha}\right)^{-1}$ is the molar weight of the multicomponent mixture. Note that Eq. (11) has been replaced by Eq. (5) in this study but is included here for completeness. According to the Stokes's hypothesis which ignoring the bulk viscosity, the shear-stress tensor for a Newtonian fluid is calculated as:

$\tilde{\tau}_{i j}=\bar{\rho} \nu(\tilde{T})\left(2 \tilde{S}_{i j}-\frac{2}{3} \delta_{i j} \tilde{S}_{k k}\right)$

where $\nu$ is a temperature-dependent kinetic viscosity, and the rate-of-strain tensor of the resolved scales is calculated as:

$\tilde{S}_{i j}=\frac{1}{2}\left(\frac{\partial \tilde{u}_{i}}{\partial x_{j}}+\frac{\partial \tilde{u}_{j}}{\partial x_{i}}\right)$

The subgrid-scale (SGS) stresses $\left(\tau_{i j}\right)$ and turbulent fluxes $\left(\Psi_{T, j}\right.$ and $\left.\Psi_{\alpha, j}\right)$ in Eq. (9) (11) are unclosed and both require specific modeling. The SGS stress defined as $\tau_{i j}=\bar{\rho}\left(\widetilde{u_{i} u_{j}}-\tilde{u}_{i} \tilde{u}_{j}\right)$, is modeled by the Boussinesq eddy viscosity hypothesis, where the SGS stresses are also taken to be proportional to $\tilde{S}_{i j}$,

$\tau_{i j}=\underbrace{\left(\tau_{i j}-\frac{1}{3} \delta_{i j} \tau_{k k}\right)}_{\text {deviatoric }}+\underbrace{\frac{1}{3} \delta_{i j} \tau_{k k}}_{\text {isotropic }}=-\bar{\rho} \nu_{\text {sgs }}\left(2 \tilde{S}_{i j}-\frac{2}{3} \delta_{i j} \tilde{S}_{k k}\right)+\frac{2}{3} \delta_{i j} \bar{\rho} k_{t}$

where $\nu_{\mathrm{sgs}}$ is the eddy viscosity given by the specified turbulence model, $k_{\mathrm{t}}$ is the unresolved turbulent kinetic energy.

The turbulent enthalpy flux term $\Psi_{T, j}=\bar{\rho}\left(\widetilde{u_{j} H_{t}}-\tilde{u}_{j} \tilde{H}_{t}\right)$ is modeled by the gradient diffusion assumption as

$\Psi_{T, j}=-2 \bar{\rho} \frac{\nu_{\text {sgs }}}{P r_{t}} \frac{\partial \tilde{H}_{t}}{\partial x_{j}}$

where $\operatorname{Pr}_{t}$ is the turbulent Prandtl number. The turbulent species diffusion term $\Psi_{\alpha, j}=\bar{\rho}\left(\widetilde{u_{j} Y_{\alpha}}-\tilde{u}_{j} \tilde{Y}_{\alpha}\right)$ is also modeled using the gradient diffusion assumption as

$\Psi_{\alpha, j}=-2 \bar{\rho} \frac{\nu_{s g s}}{S c_{t}} \frac{\partial \tilde{Y}_{\alpha}}{\partial x_{j}}$

where $S c_{t}$ is the turbulent Schmidt number. Unity $\operatorname{Pr}_{t}$ and $S c_{t}$ are used in this study.

The thermodiffusion (Soret effect), barodiffusion, and mass-driven diffusion of heat (Dufour effect) are ignored in the Favre-averaged species and energy equations Eqs. 10 and 11. In the Favre-average species Eq. (10), the SGS species diffusive flux $\frac{\partial}{\partial x_{j}}\left(\bar{\rho} \widetilde{D_{\alpha} \frac{\partial Y_{\alpha}}{\partial x_{j}}}-\bar{\rho} D_{\alpha} \frac{\partial \tilde{Y}_{\alpha}}{\partial x_{j}}\right)$ is neglected. In the Favreaveraged energy Eq. (11), the SGS energy diffusive flux $\frac{\partial}{\partial x_{j}}\left(\bar{\rho} \widetilde{D_{T} \frac{\partial H_{t}}{\partial x_{j}}}-\bar{\rho} D_{T} \frac{\partial \tilde{H}_{t}}{\partial x_{j}}+\sum_{\alpha=1}^{L}\left(\bar{\rho} D_{\alpha} \widetilde{\frac{\partial Y_{\alpha}}{\partial x_{j}}} H_{\alpha}-\bar{\rho} D_{\alpha} \frac{\partial \tilde{Y}_{\alpha}}{\partial x_{j}} \tilde{H}_{\alpha}\right)\right)$, and SGS viscous dissipation term $\frac{\partial}{\partial x_{j}}\left(\widetilde{\tau_{i j} u_{i}}-\tilde{\tau}_{i j} \tilde{u}_{i}\right)$ are also neglected. 
A hydrogen/oxygen chemical mechanism with 9 species and 19 reversible reaction steps [38,39], which has been validated over a wide pressure range $(0.3-87 \mathrm{~atm})$, is used to describe the flow properties and combustion chemistry. Accurate fluid properties are crucial to reproduce the flow behaviors. Thermodynamic properties are obtained from the NIST-JANAF thermophysical database [40]. Transport properties of the gas mixture, such as viscosity, thermal and mass diffusivities are calculated using the chemical kinetics package CHEMKIN-II [41] based on a CHEMKIN-format transport database. The mixture thermal diffusivity is calculated based on the conductivity and specific heat. Viscosity and thermal conductivity are averaged by the modified Wilke's law [42] and the combination averaging, respectively. Mass diffusivities are also mixture-averaged, and the mass conservation is achieved by setting nitrogen as the inert gas.

\section{Turbulence closure}

The Boussinesq hypothesis assuming isotropic dissipation is used to relate unresolved turbulent stresses to the rate of strain of the resolved velocity field by specifying the SGS viscosity in LES. Gradient diffusion models parameterized by constant turbulent Prandtl $\left(\operatorname{Pr}_{t}=1.0\right)$ and Schmidt $\left(S c_{t}=1.0\right)$ numbers are used to account for the heat and mass fluxes due to unresolved turbulent eddies. In this study, closure of the SGS stress tensor and scalar fluxes is accomplished by specifying an eddy viscosity $\nu_{\text {sgs }}$ calculated from the SGS turbulent kinetic energy $k_{\mathrm{sgs}}$,

$\nu_{\mathrm{sgs}}=\mathrm{C}_{k} k_{\mathrm{sgs}}^{1 / 2} \bar{\Delta}$

where $C_{k}$ is a constant, $\bar{\Delta}$ is the SGS filter width equal to the local grid dimension $\mathrm{V}^{1 / 3}$ with $\mathrm{V}$ the cell volume, $k_{\text {sgs }}$ is modeled by the dynamic subgrid kinetic energy model (DKEM) [43] to better capture the non-equilibrium kinetic energy transfer in the jet shear layer under high thermal expansion rate,

$\bar{\rho} \frac{\partial k_{s g s}}{\partial t}+\overline{\bar{\rho}} \widetilde{\vec{U}} \cdot \nabla k_{s g s}=\nabla \cdot\left(\bar{\rho} D_{e f f} \nabla k_{s g s}\right)-\tau_{i j} \cdot \nabla \widetilde{\vec{U}}-C_{\varepsilon} \frac{\bar{\rho} k_{s g s}^{3 / 2}}{\bar{\Delta}}$

where $D_{\text {eff }}$ is the effective diffusivity. The model coefficients $C_{k}$ and $C_{v}$ are determined dynamically $[44,45]$ based on the hypothesis that there is a significant correlation between the SGS stress $\tau_{i j}$ and the subtest-scale Leonard stress $L_{i j}$ [46]. Comparative studies of the performance of different SGS models [47-50] show that the k-equation model has advantages in modeling non-equilibrium turbulent dissipation and capturing fine-level fluctuation properties compared to algebraic models (e.g., Smagorinsky model [51]).

\section{Solver}

The modeling is performed by the compressible reacting flow solver AstroFoam, which is developed on the basis of the compressible flow solver rhoCentralFoam distributed with open-source CFD package OpenFOAM [52] mainly through adding the features of multi-species transport and multicomponent reaction. Similar solvers developed based on rhoCentralFoam by different research groups [53-55] have been applied in the LES modeling of supersonic combustion. AstroFoam, together with the original rhoCentralFoam solver, was firstly validated for various frozen flows, including the canonical shock tube problem, forward step flow, hypersonic flow over a biconic and supersonic jets [50,56-58]. The solver is then applied for various scramjet combustor cases $[8,21,59-64]$ to examine its accuracy and robustness in the engineering modeling of supersonic combustion.

In this study, the nonlinear inviscid convective fluxes are evaluated by the combination use [65] of a second-order semidiscrete central Kurganov-Tadmor (KT) scheme [66] and the second-order central scheme in the skew-symmetric form [67] to reduce the numerical dissipation in subsonic regions. A third-order spatial accuracy in reconstructing primitive convective fluxes at faces is achieved by the scale-selective discretization (SSD) scheme [68]. The temporal integration is advanced by the second-order Crank-Nicholson scheme $[69,70]$.

\section{Test case and numerical setup}

The test supersonic combustion case has been modeled by Lu and Jin [71-73] using DNS. The test case models a co-flowed fuel jet issued from a round orifice with a diameter of $\mathrm{D}=1.44 \mathrm{~mm}$. The fuel stream consists of $85 \% \mathrm{H}_{2}$ and $15 \% \mathrm{~N}_{2}$ by volume and has a uniform temperature of $305 \mathrm{~K}$. The fuel jet speed is $904 \mathrm{~m} / \mathrm{s}$, which corresponds to a Mach number (Ma) of 1.2 and a Reynolds number of $2.2 \times 10^{4}$. The air coflow has a subsonic speed of $20 \mathrm{~m} / \mathrm{s}$ and was heated to $1150 \mathrm{~K}$ to facilitate the ignition of the fuel.

The computation domain is a three-dimensional rectangular geometry in the dimensions of $-6 \mathrm{D}<\mathrm{x}, \mathrm{z}<6 \mathrm{D}, 0<\mathrm{y}<17 \mathrm{D}$, with the jet axis along the $y$-direction. The mesh is generated by the Cartesian CutCell method [74], which produces highquality uniform hexahedral grid cells for more than $98 \%$ of the domain volume in this study, while tetrahedron, wedge, and pyramid cells are filled only in large-curvature regions, e.g., those near the circular injector. A total of five mesh resolutions with cell numbers from $32.47,47.88,64.57,83.89$, to 106.23 million are tested. The mesh is clustered around the hydrogen jet and then stretched towards the lateral boundaries in the lateral and spanwise directions ( $\mathrm{x}$ - and $\mathrm{z}$-directions). The smallest cells, which are located on the jet axis, are $64 \mu \mathrm{m}$, which is eight times the smallest Kolmogorov scale estimated to be $8 \mu \mathrm{m}$ occurred at $\mathrm{y}=5 \mathrm{D}$ on the axis. The largest cells located near the lateral walls vary from $128 \mu \mathrm{m}$ to $256 \mu \mathrm{m}$ in the tested meshes.

As in the DNS configuration [75], fixed pressure, temperature, and velocity on the inlets of hydrogen jet (101,325 $\mathrm{Pa}$, $305 \mathrm{~K}, 904 \mathrm{~m} / \mathrm{s})$ and air coflow $(1150 \mathrm{~K}, 20 \mathrm{~m} / \mathrm{s})$ are set, while zero-gradient pressure condition is set for the subsonic air coflow inlet. Both the jet and air flows are initially in laminar status in the DNS calculation, thus zero turbulent intensity is set on both the inlets. The open boundary condition is applied to the outlet, where the zero-gradient condition is used for the outflow, while fixed ambient flow properties as the same as the coflow are specified for temperature and gas compositions in the case of return flow. In order to minimize the influence of the outlet boundary, the computational domain has been expanded in the streamwise direction from $15 D$ in the DNS 
calculation to $17 \mathrm{D}$ in the current LES modeling. A constant total pressure of $1 \mathrm{~atm}$ is set on the lateral boundary, which allows both an outflow or an entrainment inflow through. The velocity on the lateral boundary is applied as a zero-gradient condition for outflow and obtained from the patch-face normal component of the internal-cell value for inflow. The temperature on the lateral boundary is set as zero-gradient for the outflow and the coflow temperature for the inflow.

In the implementation of DZFM, there can be two types of zone division methods. One is to discretize the computation domain according to the physical coordinate as in CMC, and the other is to dynamically divide the domain according to the local flow conditions. In this study, the dynamic zone division based on both the time-variant mixture fraction fields and the physical coordinate is used. The zone is firstly uniformly divided into a finite number of swathes along the streamwise direction with a uniform height. Then each swath is further divided into 91 bins according to the local mixture fraction. The zone division is updated every 1000 time steps (approximately $\approx 7 \mu \mathrm{s}$ ) with the local flow conditions.

The computations are performed in parallel at the national supercomputer center in Tianjin (TH-1) using $280 \mathrm{CPU}$ cores (Intel(R) Xeon(R) CPU E5-2690v4 with the base frequency of $2.60 \mathrm{GHz}$ ). The time step is limited both by a maximum Courant number of 0.5 and a user-specified maximum time step of $1 \times 10^{-8} \mathrm{~s}$. The typical time step in the modeling is $7 \times 10^{-9} \mathrm{~s}$. The flow-through time (FTT) based on the inlet jet velocity and the stream-wise domain length is $27 \mu$ s? The modeling was advanced with 20 FTTs for data sampling and statistics. The modeling based on the finest mesh costs around 1,424, $\times 280$ CPU hours, which is 50 times less than the DNS modeling based on 975 million cells [75].

\section{Results and discussion}

\section{Validation in a supersonic reacting jet}

Fig. 1 compares the predictions of mean axial velocity under different mesh resolutions with the DNS data [75]. The agreements are generally good, except that the coarsest mesh (32.47 million cells) slightly overpredicts the axial velocity. Compared with the DNS data, two observable differences exist near the jet entrance and the outlet. The initial compression and expansion process in the current LES modeling is not observed in the DNS modeling, which could be due to different configurations of the inlet velocity, e.g., using a top-hat profile. Since no additional information is provided for the setup of the inlet velocity profile, no top hat profile has been configured in the current modeling. Due to the large velocity difference, the strong initial shear stress in the jet boundary produces a small oscillation in the near-field pressure. Nevertheless, such small variation $(<0.2 \%)$ is considered to have little influence on subsequent jet development. The sudden rise of velocity in the far downstream near the outlet in the DNS modeling is expected to be artificially caused by the outlet boundary condition. Theoretically, the axial velocity should always decay due to the viscous effect if without any external forces(s) (like buoyancy). The rise of axial velocity in the last state in the DNS calculation indicates that an artificial suction force has been produced on the outlet. However, duo the hyperbolic flow feature of supersonic flow, the adverse influence of the outlet cannot vitiate the upstream flow. In order to minimize the influence of the outlet boundary, the computational domain in the current modeling has been extended in the streamwise direction for an additional distance of $2 D$. Since no experimental data are available, the relative errors are calculated in reference to the finest LES modeling with 106.23 million cells, as $\left|\mathrm{U}-\mathrm{U}_{106.23 \mathrm{M}}\right| \mathrm{U}_{106.23 \mathrm{M}}$. From the logarithmic diagram in Fig. 1 (b), both the mean and maximum errors decrease almost linearly as the mesh is refined. The relative errors are all smaller than $2.2 \%$, which satisfies the requirement of grid independence. In the following analysis, the results based on the finest mesh are analyzed.

Fig. 2 (a) compares the peak temperature in the shear/ mixing layer by discretizing the whole field into different numbers of equal-width swathes in the streamwise direction while maintaining the same discretization number in the mixture fraction space ( 91 points). The temperature profiles deviate considerably from the others when treating the streamwise domain as an ensemble $(1 \times 91$ zones $)$ and as three swathes $(3 \times 91$ zones). Under fine axial discretizations with more than 61 swathes, the temperature profiles are generally similar to each other. As the zone shrinks, those zone-sampled parameters (e.g., for scalar dissipation rate, temperature, and pressure) can represent the local mixing and chemical status more accurately since their spatial variations
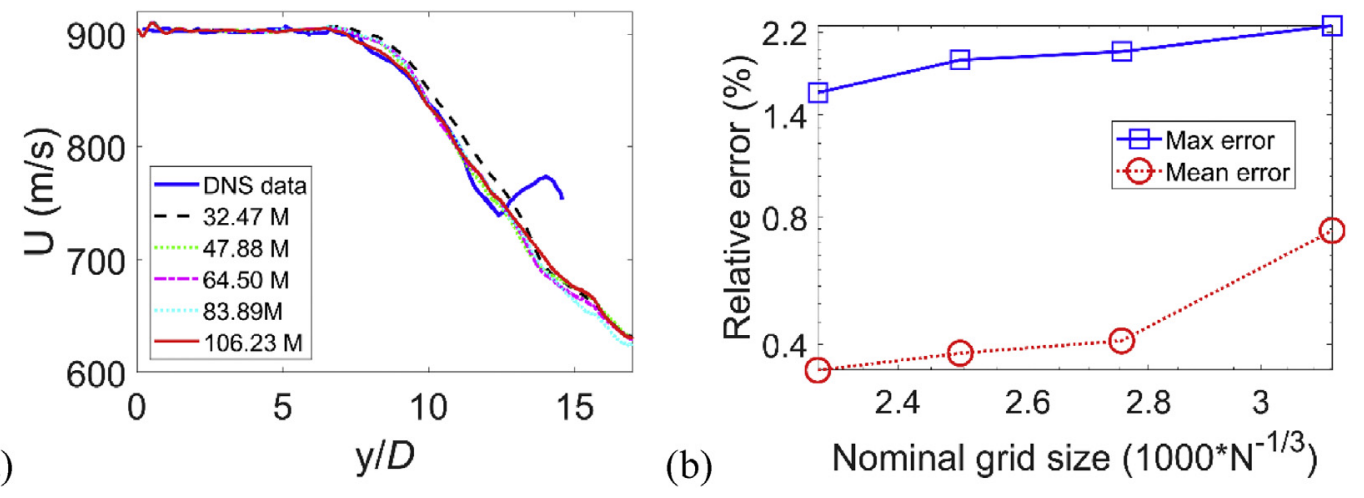

Fig. 1 - (a) Comparison of axial profiles of mean velocity, and (b) grid convergence analysis. 


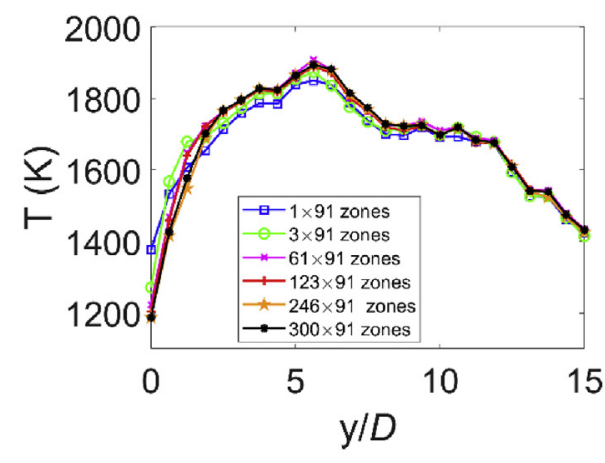

(a)

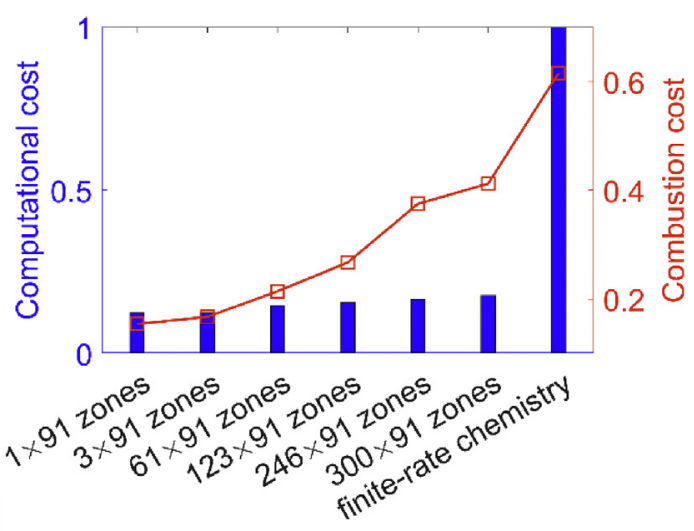

(b)

Fig. 2 - (a) Temperature dependence on the zone discretization, and (b) the computational cost scaled by that of the finiterate chemistry modeling, and the combustion modeling time scaled by the entire computational time under different zone discretizations.

have been effectively truncated. However, when the flamelet zone spans a wider range in the streamwise direction, the operation of zone average and zone-based conditional average would smear the difference between the upstream and downstream reacting regions. For example, the upstream mixture will be artificially ignited by the downstream burnt mixture since they are within the same zone and share the same flamelet, as observed from the higher initial temperature for $1 \times 91$ zones and $3 \times 91$ zones in Fig. 2 (a). The initial temperature with $61 \times 91$ zones and $12,3 \times 91$ zones is also slightly higher than that in the two finest-zone cases. The wide spatial spanning of the flamelet zone also tends to produce a zone average or zone-based conditional average considerably lower than the peak value since the median value is always selected, e.g., a lower representative temperature. Correspondingly the reacting progress under the control of the representative temperature has obviously been slowed down for $1 \times 91$ zones and $3 \times 91$ zones as indicated by the lower flame temperature in Fig. 2 (a) For the two finest zone case, the almost identical profiles indicate that the zone convergence has been achieved. In the following analysis, the division with $30,0 \times 91$ zones will be used if not specified otherwise.

As the zone division refines, the computational cost (denoted by computational time) for the combustion modeling part increases drastically as more zone flamelets need to be resolved, while the flow modeling part remains nearly the same cost. However, even with the finest zone division, the entire computational cost is still small compared with the finite-rate chemistry modeling with full species transport. The computational cost of DZFM calculations in Fig. 2 (b) has been scaled by the cost of the finite-rate chemistry modeling, and the DZFM calculation with $30,0 \times 91$ zones takes only $17.7 \%$, even less than $1 / 5$ of the cost of the finite-rate chemistry modeling. The ratio of combustion modeling time to the entire computational time increases almost linearly with the zone discretization number, from $15.4 \%$ for $1 \times 91$ zones to $41.2 \%$ for $30,0 \times 91$ zones, while more than half, $61.5 \%$ of the cost is spent in the finite-rate chemistry modeling. The computational cost also increases significantly due to the resolving of all the species transport equations in finite-rate chemistry, while only two equations for mixture fraction and its variance are solved in DZFM.

Figs. 3 and 4 compare the radial distributions of mean velocity and temperature at different axial heights. Here $\delta_{1 / 2}$ refers to the jet width defined by the radial distance where the velocity is half of that on the axis. The current predictions of radial velocity profiles are in good agreement with the DNS data, with only minor differences near the jet boundary. The "smearing" effect on the jet boundary in the LES modeling could be overcome by further refining the mesh towards the implicit LES or DNS level through reducing the numerical dissipation to enable a sharper jet boundary capture. The radial temperature profiles are also in reasonable agreement with the DNS data. Compared with the traditional flamelet model based on a single flamelet, the current zone-based flamelet modeling offers a more accurate description of the local chemical status. In the stage of $y=0-1 D$, the initial reactions produce relatively low temperature, which has been well reproduced by using a fine streamwise zone discretization. The local flamelet evolves from a pure mixing status succeeded from the inlet boundary flux. As the jet flows from $O D$ to $1 D$, the mixture in the mixture fraction space reacted under the same flow residence time, thus mimicking the ignition process in the real flow. Subsequently, the flamelet at $y=1 D$ is transported to the downstream $y=2 D$ with the reactions continue in the mixture fraction space. The radial temperature profile is successfully reproduced at $y=2 D$, indicating that the flamelet there well represents the local composition status versus the mixture fraction. At $y=4 D$, the peak temperature in the DNS modeling has a sudden drop, whereas the current LES prediction does drop but at a lower level, as also indicated in Fig. 2 (a). Lu [76] explained that the lower temperature is due to suddenly enhanced mixing with the colder jet flow after the natural transition at around $y=3 D$. However, the prediction of such laminar-to-turbulent transition behavior falls out the capability of the current explicit LES model. In this study, the jet flow starts from a very weak turbulence status to mimic the initial laminar status, but a more accurate prediction of the turbulent evolution may require a more delicate approach like implicit LES based on a finer mesh. The radial temperature profile at $y=8 D$ is also 

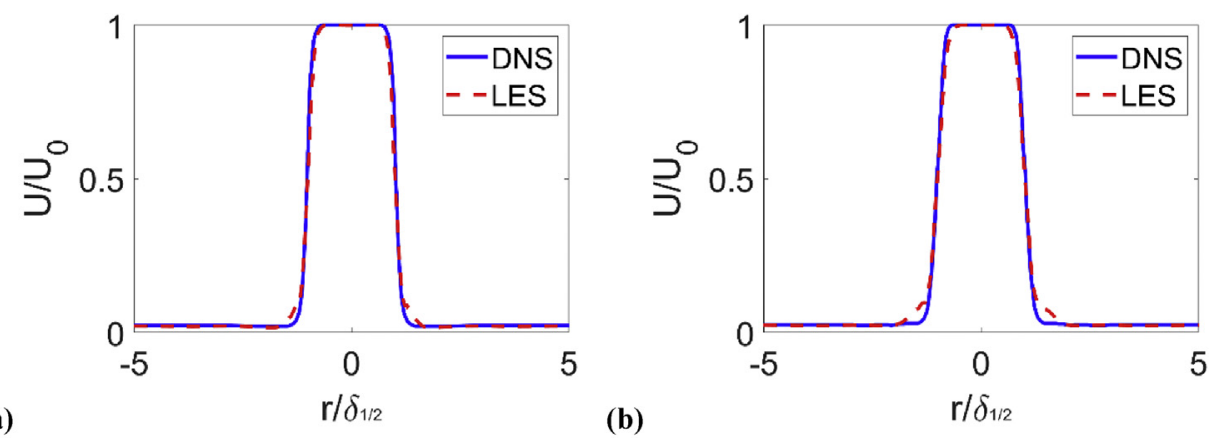

(a)

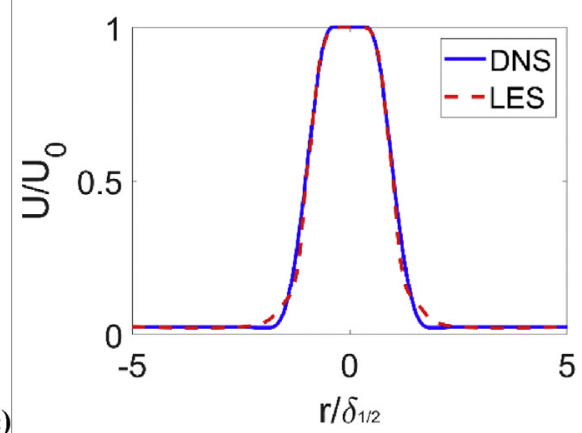

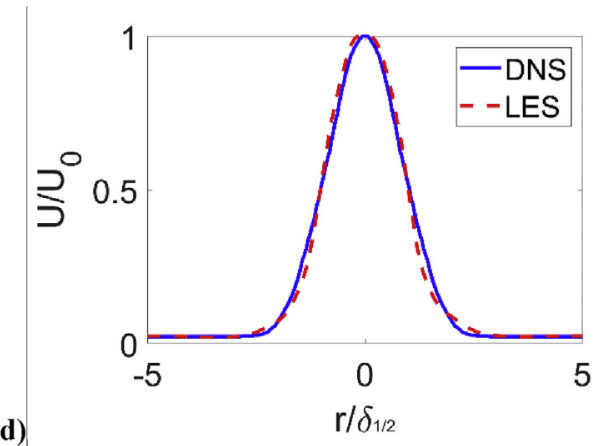

Fig. 3 - Comparison of radial profiles of mean velocity at (a) $y=1 D$, (b) 2D, (c) 4D, and (d) 8D.
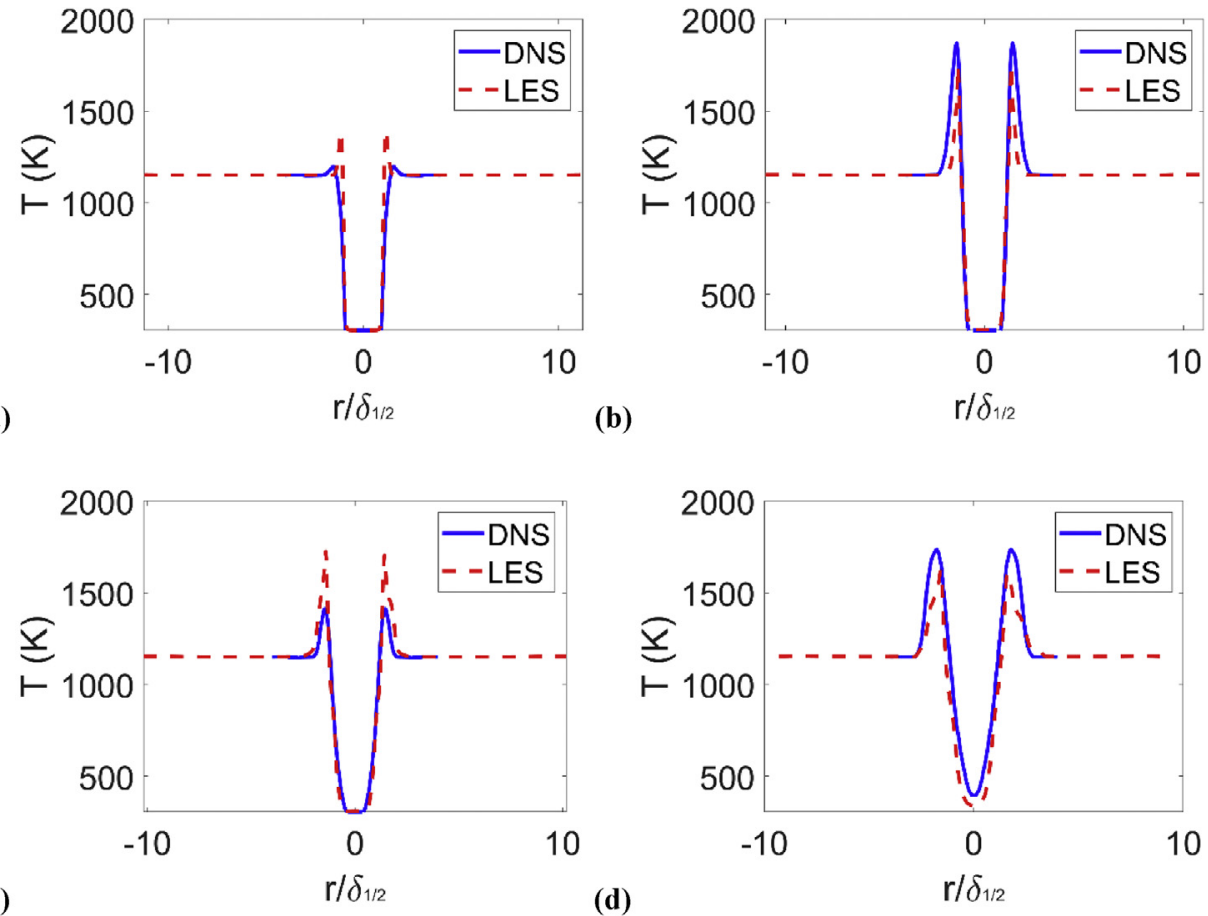

Fig. 4 - Comparison of radial profiles of mean temperature at (a) $y=1 D$, (b) $2 D$, (c) $4 D$, and (d) $8 D$.

comparable with the DNS prediction, indicating that the reaction progress from $0 D$ to $8 D$ has been reasonably reproduced in the evolvement of the flamelet while being transported from the upstream to the downstream.

In Fig. 5, the instantaneous flame is presented as the contours of temperature overlaid by the vortex structures and the mass fraction of $\mathrm{H}_{2} \mathrm{O}$. As stated in the DNS study [75], the jet is lifted and auto-ignited, which has been successfully reproduced in the current zone-based flamelet modeling. If registering ignition when the mean temperature reaches a value of $1400 \mathrm{~K}$, which indicates an obvious temperature rise of $250 \mathrm{~K}$ above the initial oxidizer temperature, the flame lift-off distance is determined to be $0.8 \mathrm{D}$, which is comparable to the DNS prediction of $0.86 D$ [75]. While in the ensemble flamelet 


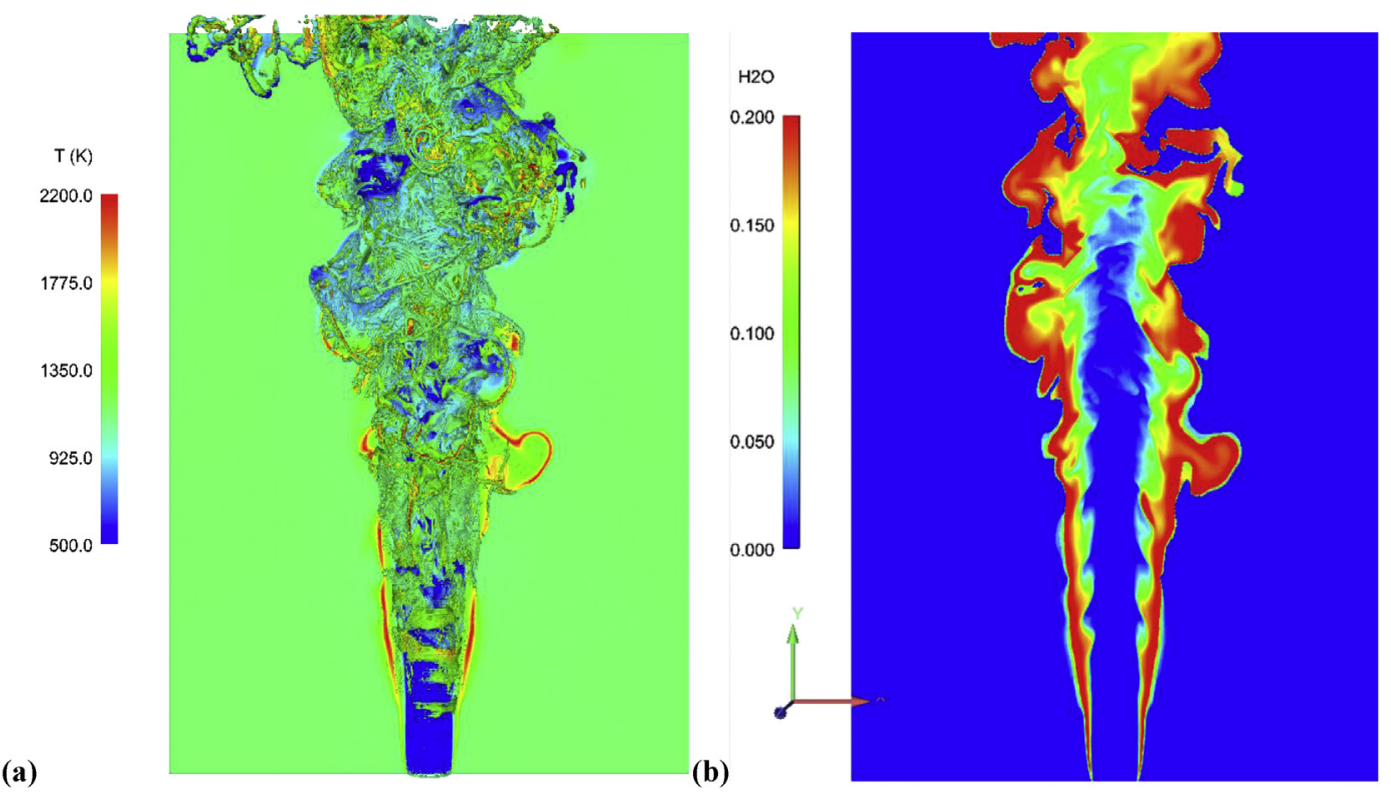

Fig. 5 - (A) Vortex structure represented by the iso-surfaces of Q-criterion (the second invariant of the velocity gradient tensor) at $2 \times 10^{10} \mathrm{~s}^{-2}$ and colored by static temperature, (b) flame structure represented by the contour of $\mathrm{H}_{2} \mathrm{O}$ mass fraction.

case $(1 \times 91$ zones), the flame is artificially ignited at the very beginning (with nearly zero lift-off distance) by the downstream burnt status as denoted in Fig. 2 (a). The high temperature mainly occurs in a thin layer attached to the jet core. At around $y=4 D$, an intermittence in the high-temperature layer can be observed as in the DNS calculation [72], which is reflected as the slightly lower temperature in Fig. 4(c). A single flamelet is incapable of reproducing such an ignition process and the lifted flame base, as the downstream equilibrium chemical status would artificially ignite the upstream mixture. The gradual erosion of the $\mathrm{H}_{2} \mathrm{O}$ contour into the jet core with the increasing of the axial height in Fig. 5 (b) exhibits the establishment of a fully mixing and complete burning status towards the outlet. As shown in Fig. 5 (a) and (b), at $\mathrm{y}=1.5 \mathrm{D}$ and $\mathrm{y}=12 \mathrm{D}$, the shear/mixing layer has two sudden thickening; the former is due to the formation of ring vortexes, while the latter is due to the breakdown of the ring and streamwise vortexes into fine vortexes.

Fig. 6 shows the time-variation of the flamelet zones, which are dynamically adapting to the short-time averaged mixture fraction field. In the current zone division strategy, the turbulent fields are equidistantly cut into a finite number of swathes along the streamwise direction (+y direction), then the swathes are further cut into a finite number of annual rings according to the local mean mixture fraction, as though the tree rings on a stump. The mixture fraction distribution varies significantly in the violent mixing region, thus the zone division needs to be updated more frequently in the downstream region. Note that the zone division also varies with the axial height since the mixture fraction distribution varies. Through constantly adapting the zone division with the timevariant mixture fraction field, each flamelet zone corresponds to a small mixture fraction subspace. If the mixture fraction subspace of the current flamelet zone does not correspond to any subset of the mixture fraction field, the current flamelet zone will be "frozen" temporally. For example, at the current shown downstream location of $y=17 D$, the fuel-rich mixture fraction range vanishes due to the dispersion, thus only those flamelet zones corresponding to fuel-lean conditions are activated. Similarly, in the upstream, the mixture fraction distribution is much concentrated, thus only a few numbers of flamelet zones corresponding to the extremes of fuel-rich and oxygen-rich conditions are activated. The zone flamelet equation for those "frozen" zones is not solved as no CFD cells have been assigned to them, and therefore all the zone-based or zone-statistical variables (like representative temperature) become meaningless. Accordingly, the computational cost for those "frozen" zones can be saved, with only a minimum memory cost. However, those "frozen" zones have the probability of being re-activated, and the frozen chemical status will be updated once again in a future time step, remembering that the zone division is constantly updating with the timevariant mixture fraction field. As seen, the word "frozen" means that a flamelet is assigned to a void zone in the current zone division, but the flamelet must be retained due to the reuse possibility of the stored information in the "frozen" flamelet. The zone can be in irregular shape and contains a random number of CFD cells. Note that the flamelet zone does not need to be continuous, but can be composed of several isolated "islands", as the case in Fig. 6 (a), as long as their local mixture fraction falls into to the mixture fraction subspace that is allocated to the flamelet zone.

Figs. 7 and 8 show the status of temperature and $\mathrm{H}_{2} \mathrm{O}$ concentration in the flamelet zones at different axial heights and corresponding to the stoichiometric mixture fraction. Note that the representative temperature is not directly solved from the flamelet energy equation but is reversely obtained from the flow fields using the statistical enthalpy approach. The representative temperature determines the reaction rates within each mixture fraction bin for the flamelet assigned to 
(a)
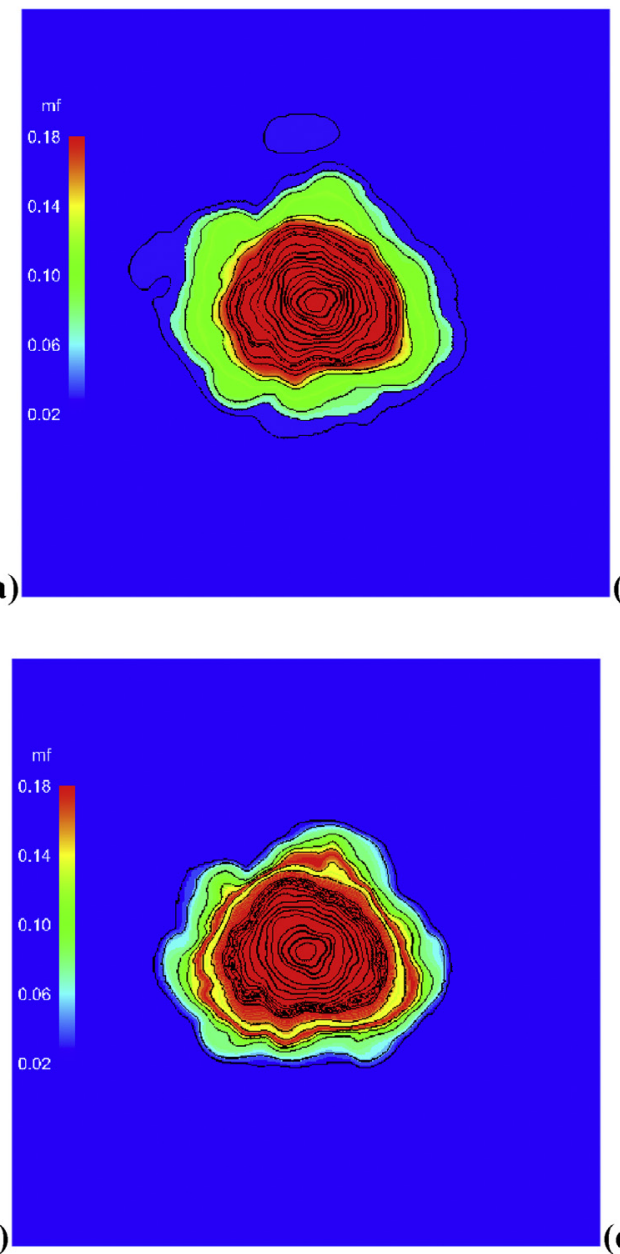

(c)

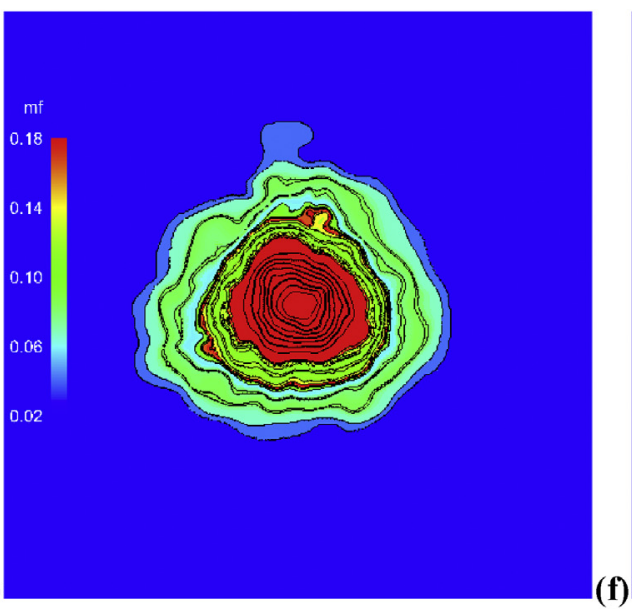

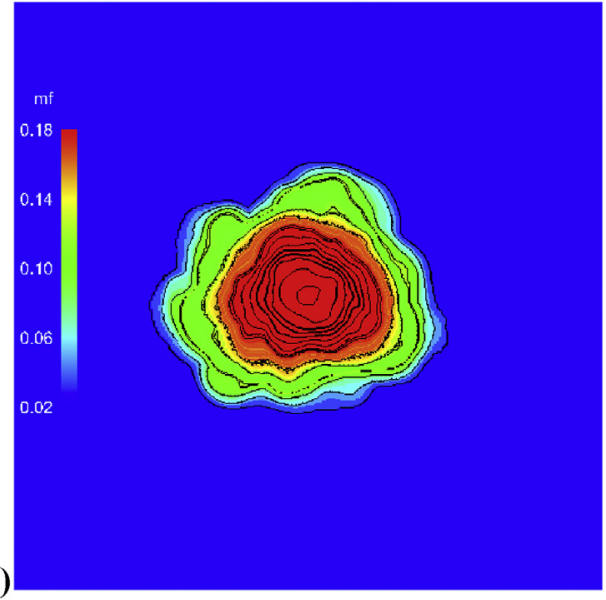

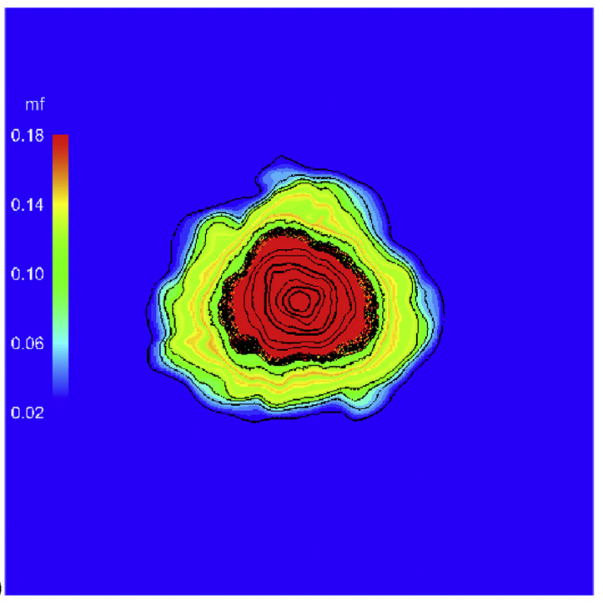

(d)

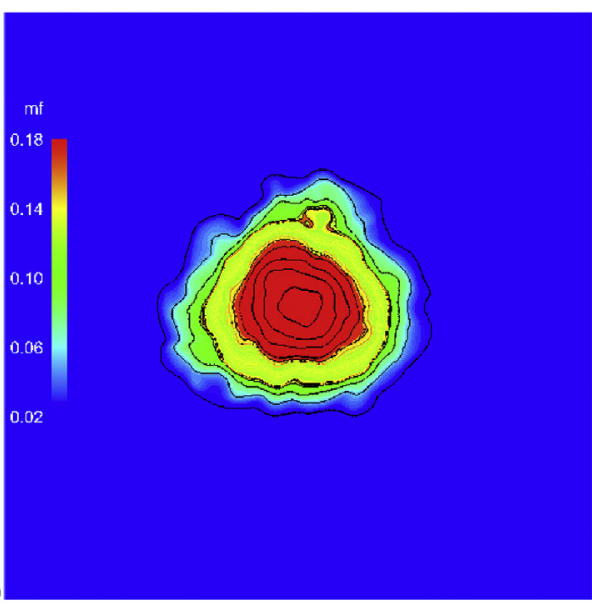

Fig. 6 - Time evolution of dynamic adaptive zones at $y=17 \mathrm{D}$ with an interval of $1 \times 10^{-4} \mathrm{~s}$.

the current zone. In the flamelet zones attached to an inflow boundary, the conditional species flux is replenished in the pure mixing status through the zone boundary overlapping with the inflow boundary. In the flamelet zones attached to an outflow boundary, a zero-gradient boundary condition is applied for the outflow conditional species flux. The chemical status in one flamelet zone is succeeded by its neighbor zones if the mass flux across the zone boundary between them is in the direction from the current zone to the neighbor zone. The flamelet is then updated as the summation of the imported and current flamelets weighted by imported mass and the current zone-owned mass. The current zone division strategy along the streamwise direction implies that the flamelets evolve physically from the unburnt mixing status in the lowest zones to the burnt status in the downstream zones, under the control of different representative temperature 


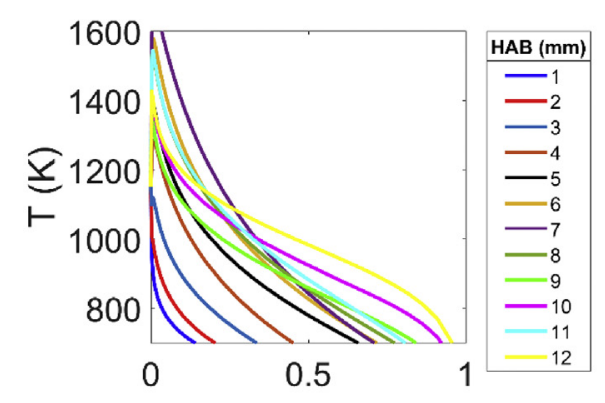

(a)

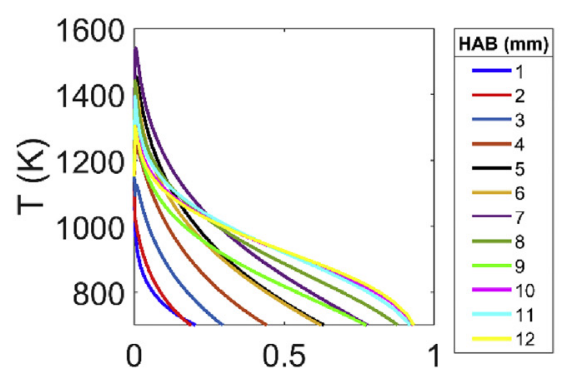

(c)
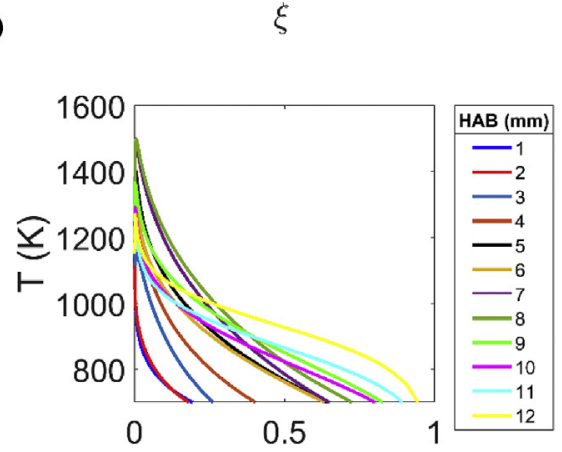

(e)

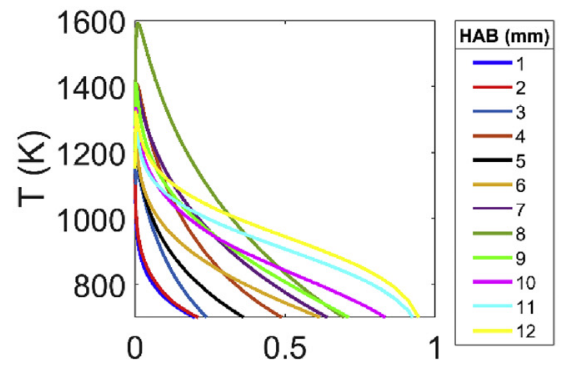

(g)
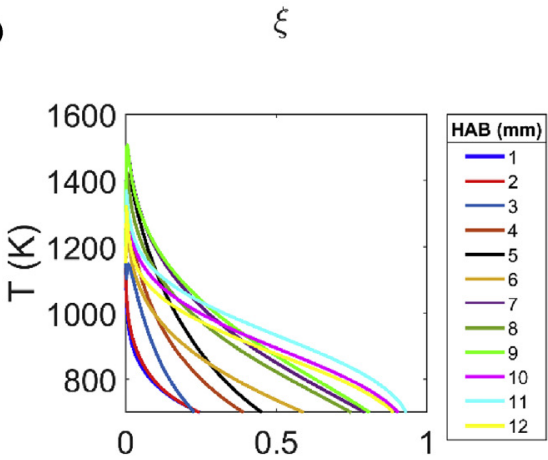

(i)

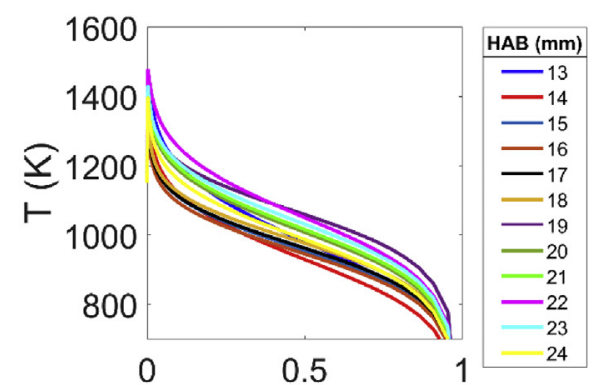

(b)

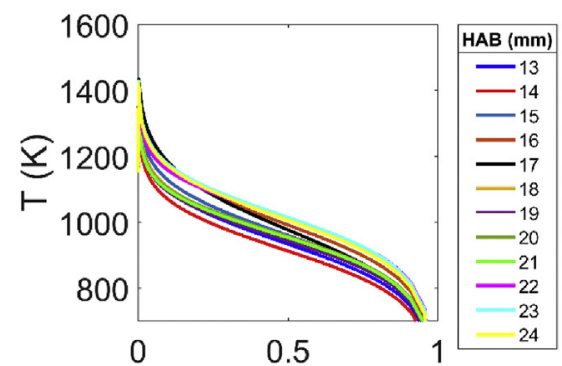

(d)

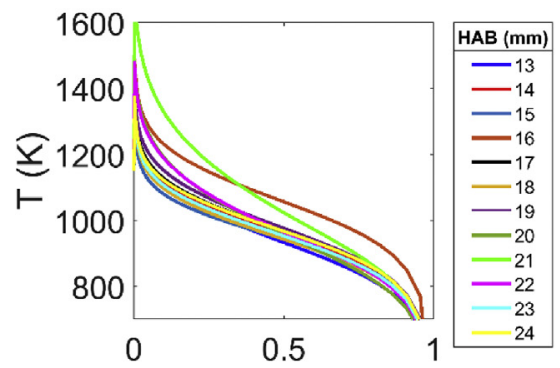

(f)

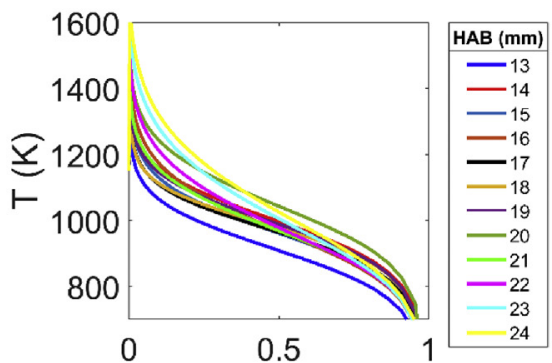

(h)

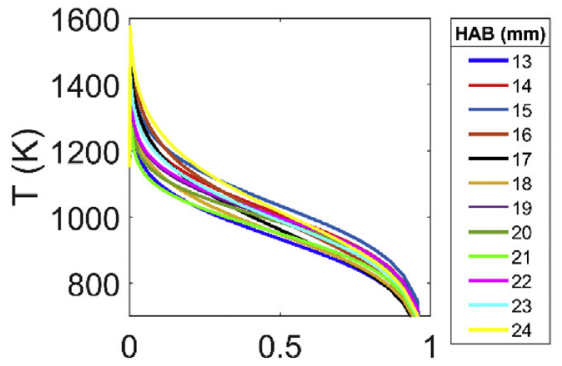

(j)

$\xi$

Fig. 7 - Representative temperature in the mixture fraction space at different HABs and subsequent times with an interval of $2 \times 10^{-5} \mathrm{~s}$. 


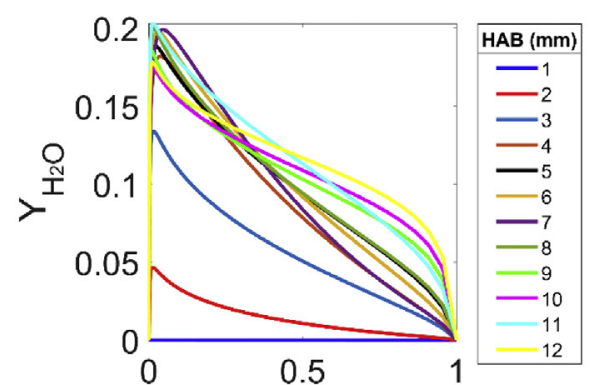

(a)

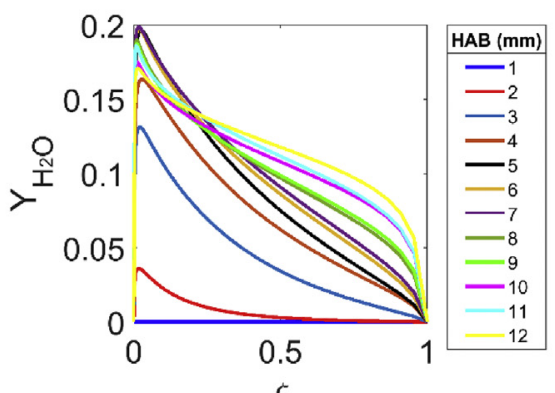

(c)

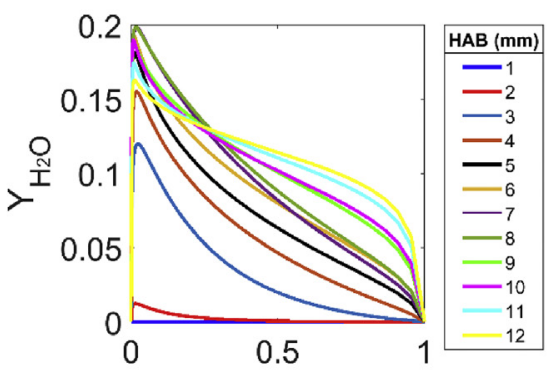

(e)

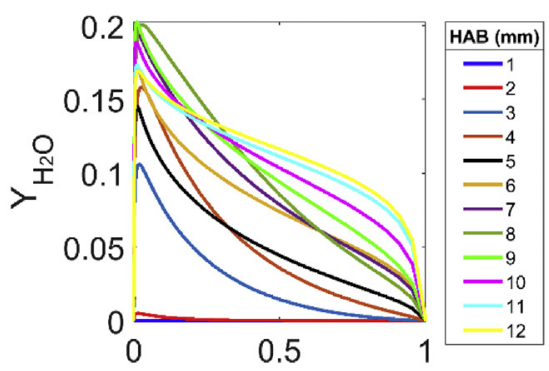

(g)

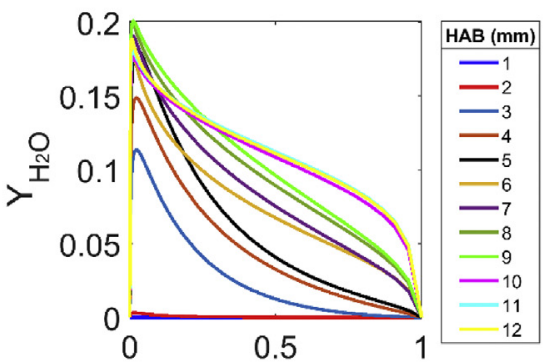

(i)

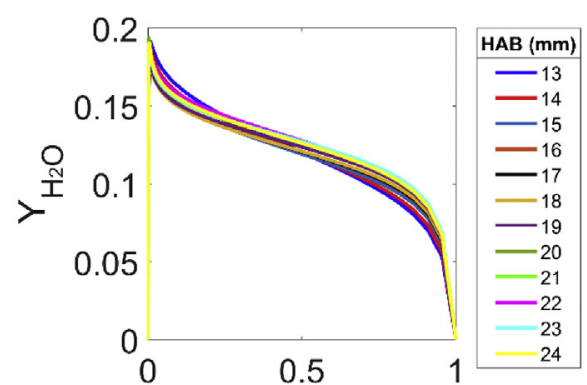

(b)

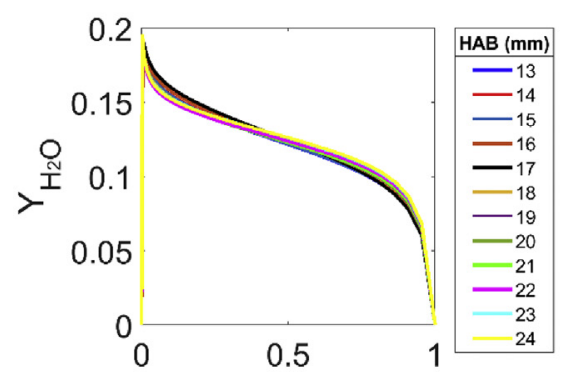

(d)

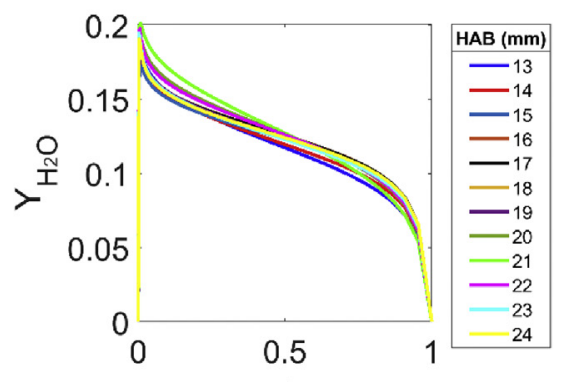

(f)

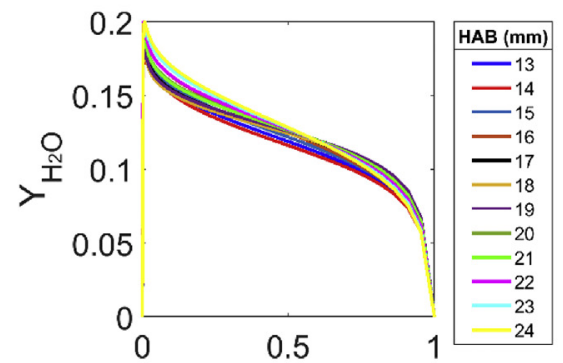

(h)

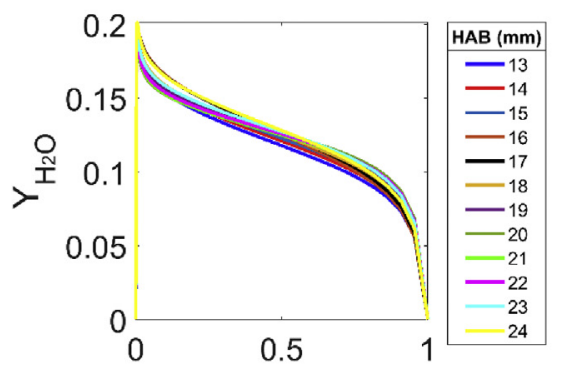

(j)

Fig. 8 - Representative mass fraction of $\mathrm{H}_{2} \mathrm{O}$ in the mixture fraction space at different $\mathrm{HABs}$ and subsequent times with an interval of $2 \times 10^{-5} \mathrm{~s}$. 
when through different zones. The representative temperature and the concentration of the main product $\left(\mathrm{H}_{2} \mathrm{O}\right)$ clearly show a gradually increasing trend as the axial height increases, which is an indication of the successful auto-ignition and the gradual progress towards the equilibrium. At the further downstream locations above HAB (height above the burner) $=13 \mathrm{~mm}$, the representative temperature and $\mathrm{H}_{2} \mathrm{O}$ concentration change only slightly with the axial height, indicating that the combustion reactions there have reached equilibrium.

Both the representative temperature and the compositions change dynamically with time because the chemical status of the mixture within each zone is constantly evolved due to the spatial exchange with its neighbors through the zone border, the chemical reactions, as well as the differential diffusion in the mixture fraction space. The diffusion of radicals across the mixture fraction space can also ignite the mixture in the surrounding mixture fraction bins, which mimics the one of the important ignition mechanism in high-speed flows due to radical farming in the physical space. The dynamic update in zone division can also alter the flamelet since different CFD cells may be contained in one flamelet zone. After updating the zone division, the new flamelet is collecting from its zone-owned CFD cells by summing the old flamelet(s) with the weight of the probability density function multiplied by the cell mass, $\varphi \mid \eta_{\text {zone, new }}=\left(\sum_{\text {cell }}\langle\varphi \mid \eta\rangle_{\text {zone, old }} \cdot \mathrm{P}(\eta) \cdot \rho \Delta_{\mathrm{V}}\right) /\left(\sum_{\text {cell }} \rho \Delta_{\mathrm{V}}\right)$, where $\Delta_{V}$ is the cell volume. As seen, both the zone division and the flamelet are dynamically updated to better represent the local chemical status in terms of mixture fraction within each flamelet zone.

The historical statistics of instantaneous temperature versus mixture fraction are shown in Fig. 9. The temperature is used because it is directly resolved from the energy transport equation rather than the integration from the flamelet libraries. Two types of zone division methods are used, i.e., a fixed zone based on the physical coordinates as in the traditional CMC implementation, and the current dynamic zone division adaptive to the mixture fraction field. Different colors are used to differentiate the data points in different zones. As seen, the data points in the fixed zone are rather scarce in the mixture fraction space and span over wider ranges of mixture fraction space. Whereas the data points in the dynamic zone cluster around the narrow mixture fraction subspace that is allocated to the zone, therefore the operation of conditional average is physically more accurate and the assumption on the conditional fluctuation terms in Eq. (5) can be safely made.

\section{Conclusions}

A dynamic zone flamelet model (DZFM) is proposed in this study to better represent the local chemical status under different turbulence-chemistry interactions and to alleviate the computational cost in supersonic modeling. The model divides the whole computational domain into a finite number of control zones with the chemical status in each zone represented by a local flamelet. Through adapting the zone division with the time-variant mixture fraction field, the scattering of variables over the mixture fraction space is in controllable small, thus the representative flamelet characterizes the real scalar distribution better. The flamelet within each zone is constantly evolved due to the spatial exchange among its neighbors through the zone border, the chemical reactions, as well as the differential diffusion in the mixture fraction space. In highly compressible supersonic flows, the density, temperature, and velocity do not correlate with the mixture fraction any more, thus only the flamelet equations for species are solved, while a statistical method is used to obtain the representative temperature within each flamelet zone. Both the zone division and the flamelet are dynamically updated to better represent the local chemical status in the mixture fraction space.

The zone-based flamelet model is then applied to the modeling of a supersonic hydrogen jet flame in the framework of high-fidelity large eddy simulation based on 106.23 million cells and 30,0 × 91 flamelet zones, whose grid and zone independence have been validated. The axial and radial velocity profiles agree well with the DNS data. The radial temperature profiles indicate that the current model well reproduces the auto-ignition process at the flame base and the reaction progress at different axial heights. The autoignition process and the flame lift-off phenomenon are well reproduced by the current model, which offers a more accurate description of the local chemical status and is superior to the traditional flamelet model based on a single flamelet. In the flamelets, the representative temperature and the concentration of the product $\left(\mathrm{H}_{2} \mathrm{O}\right)$ clearly show a gradually increasing trend as the
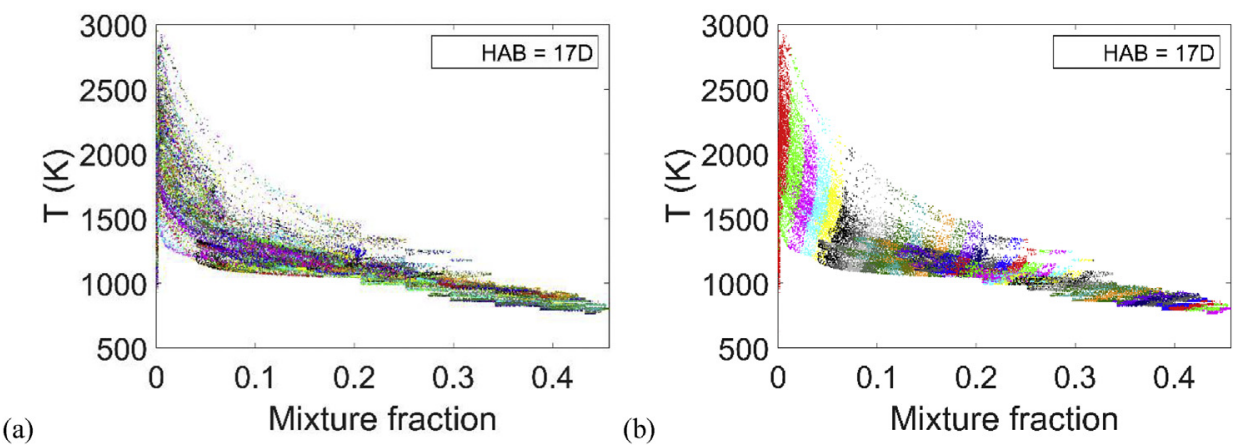

Fig. 9 - Historical statistics of temperature versus mixture fraction at $y=17 D$ for (a) fixed zone and (b) dynamic zone. 
axial height increases, which is an indication of the successful auto-ignition and the proceeding of the reaction progress towards the equilibrium. Through constantly adapting the zone division with the time-variant mixture fraction field, each flamelet zone corresponds to a narrow mixture fraction subspace. Compared with the fixed zone approach, the data points in the dynamic zone are clustering around the mixture fraction that is allocated to the zone, therefore the conditional average is physically more accurate. .The DZFM calculation with $30,0 \times 91$ zones takes only $17.7 \%$, even less than $1 / 5$ of the cost of the finite-rate chemistry modeling. The ratio of combustion modeling time to the entire computational time increases almost linearly with the zone discretization number, from $15.4 \%$ for $1 \times 91$ zones to $41.2 \%$ for $30,0 \times 91$ zones, while more than half, $61.5 \%$ of the cost is spent in the finite-rate chemistry modeling.

\section{Acknowledgments}

The project was supported by Training Program of the Major Research Plan of the National Natural Science Foundation of China (Grant No. 91641110), National Key Research and Development Program of China (2019YFB1704200), and the Open founding of National Key Laboratory of Science and Technology on Aero-Engine Aero-Thermodynamics (Grant No. 6142702180307). The authors are also grateful to the National Supercomputer Center in Tianjin for providing the computational resource.

\section{REFEREN CES}

[1] Peters N. Laminar diffusion flamelet models in nonpremixed turbulent combustion. Prog Energy Combust Sci 1984;10:319-39.

[2] Cuenot B, Egolfopoulos FN, Poinsot T. An unsteady laminar flamelet model for non-premixed combustion. Combust Theor Model 2000;4:77-97.

[3] Ingenito A, Bruno C. Physics and regimes of supersonic combustion. AIAA J 2010;48:515-25.

[4] Pitsch H, Wan YP, Peters N. Numerical investigation of soot formation and oxidation under diesel engine conditions. Toronto, Ontario: Fuels \& Lubricants Meeting \& Exposition; 1995.

[5] Kundu P, Pei Y, Wang M, Mandhapati R, Som S. Evaluation of turbulence-chemistry interaction under diesel engine conditions with multi-flamelet RIF model. Atomization Sprays 2014;24:779-800.

[6] Klimenko AY. On the relation between the conditional moment closure and unsteady flamelets. Combust Theor Model 2001;5:275-94.

[7] Huang Z, Zhang H. Numerical investigations of mixed supersonic and subsonic combustion modes in a model combustor. Int J Hydrogen Energy 2020;45:1045-60.

[8] Yao W, Wu K, Fan X. Influences of domain symmetry on supersonic combustion modeling. J Propul Power 2019;35:451-65.

[9] Curran D, Wheatley V, Smart M. Investigation of combustion mode control in a Mach 8 shape-transitioning scramjet. AIAA J 2019;57(7):2977-88.
[10] Yao W, Li B. Application of dynamic zone flamelet model to a $\mathrm{GH} 2 / \mathrm{GO} 2$ rocket combustor. Indianapolis, Indiana: AIAA Propulsion and Energy Forum and Exposition; 2019.

[11] Yao W, Fan X. In: Development of zone flamelet model for scramjet combustor modeling 21st AIAA international space planes and hypersonic systems and Technology conference. Xiamen, China; 2017.

[12] Wen JX, Xu BP, Tam VHY. Numerical study on spontaneous ignition of pressurized hydrogen release through a length of tube. Combust Flame 2009;156:2173-89.

[13] Qin F, Huang Z-w, He G-q, Wang S, Wei X-g, Liu B. Flame stabilization mechanism study in a hydrogen-fueled model supersonic combustor under different air inflow conditions. Int J Hydrogen Energy 2017;42:21360-70.

[14] Huang Z-w, He G-q, Qin F, Wei X-g. Large eddy simulation of flame structure and combustion mode in a hydrogen fueled supersonic combustor. Int J Hydrogen Energy 2015;40:9815-24.

[15] Cao C, Ye T, Zhao M. Large eddy simulation of hydrogen/air scramjet combustion using tabulated thermo-chemistry approach. Chin J Aeronaut 2015;28:1316-27.

[16] Potturi AS, Edwards JR. Large-eddy/Reynolds-averaged Navier-Stokes simulation of cavity-stabilized ethylene combustion. Combust Flame 2015;162:1176-92.

[17] Liu C, Wang Z, Wang H, Sun M, Li P. Large eddy simulation of cavity-stabilized hydrogen combustion in a diverging supersonic combustor. Int J Hydrogen Energy 2017;42:28918-31.

[18] Edwards JR, Boles JA, Baurle RA. Large-eddy/Reynoldsaveraged Navier-Stokes simulation of a supersonic reacting wall jet. Combust Flame 2012;159:1127-38.

[19] Fulton JA, Edwards JR, Cutler A, McDaniel J, Goyne C. Turbulence/chemistry interactions in a ramp-stabilized supersonic hydrogen-air diffusion flame. Combust Flame 2016;174:152-65.

[20] Klimenkoa AY, Bilger RW. Conditional moment closure for turbulent combustion. Prog Energy Combust Sci 1999;25:595-687.

[21] Yao W, Lu Y, Wu K, Wang J, Fan X. Modeling analysis of an actively-cooled scramjet combustor under different kerosene/air ratios. J Propul Power 2018;34:975-91.

[22] Yao W, Wang J, Lu Y, Li X, Fan X. Full-scale Detached Eddy Simulation of kerosene fueled scramjet combustor based on skeletal mechanism. In: 20th AIAA international space planes and hypersonic systems and technologies conference. Glasgow, Scotland; 2015.

[23] Fan Z, Liu W, Sun M, Wang Z, Zhuang F, Luo W. Theoretical analysis of flamelet model for supersonic turbulent combustion. Sci China Technol Sci 2011;55:193-205.

[24] O'Brien EE, Jiang T-L. The conditional dissipation rate of an initially binary scalar in homogeneous turbulence. Phys Fluids 1991;3:3121-3.

[25] Pera C, Reveillon J, Vervisch L, Domingo P. Modeling subgrid scale mixture fraction variance in LES of evaporating spray. Combust Flame 2006;146:635-48.

[26] Klimenko AY, Bilger RW. Conditional moment closure for turbulent combustion. Prog Energy Combust Sci 1999;25:595-687.

[27] Thornber B, Bilger RW, Masri AR, Hawkes ER. An algorithm for LES of premixed compressible flows using the Conditional Moment Closure model. J Comput Phys 2011;230:7687-705.

[28] Ladeinde F, Lou Z. Improved flamelet modeling of supersonic combustion. J Propul Power 2018.

[29] Gao Z, Jiang C, Lee C-H. Representative interactive flamelet model and flamelet/progress variable model for supersonic combustion flows. Proc Combust Inst 2017;36:2937-46. 
[30] Jesse Q, Tomasz GD, James CM, Guilhem L, Joseph CO. A priori analysis of a compressible flamelet model using RANS data for a dual-mode scramjet combustor. In: 22nd AIAA computational fluid dynamics conference. Dallas, TX: American Institute of Aeronautics and Astronautics; 2015.

[31] Zhang D, Tan J, Lv L. Investigation on flow and mixing characteristics of supersonic mixing layer induced by forced vibration of cantilever. Acta Astronaut 2015;117:440-9.

[32] Saghafian A, Terrapon VE, Pitsch H. An efficient flameletbased combustion model for compressible flows. Combust Flame 2015;162:652-67.

[33] Cook AW, Cabot WH. Hyperviscosity for shock-turbulence interactions. J Comput Phys 2005;203:379-85.

[34] Chen L, Yao W, Fan X. Influence of compressibility effect on supersonic combustion modeling. Indianapolis, Indiana: AIAA Propulsion and Energy Forum and Exposition; 2019.

[35] Jones WP, Whitelaw JH. Calculation methods for reacting turbulent flows: a review. Combust Flame 1982;48:1-26.

[36] Piomelli U. Large-eddy and direct simulation of turbulent flows. In: Jv Beeck, Benocci C, editors. Introduction to turbulence modelling. Belgium. Von Karman Institute; 2004.

[37] Sankaran V, Genin F, Menon S. Subgrid mixing modeling for large eddy simulation of supersonic combustion. In: 42nd AIAA aerospace sciences meeting and exhibit. American Institute of Aeronautics and Astronautics; 2004.

[38] Burke MP, Chaos M, Ju Y, Dryer FL, Klippenstein SJ. Comprehensive H2/O2 kinetic model for high-pressure combustion. Int J Chem Kinet 2012;44:444-74.

[39] Li J, Zhao Z, Kazakov A, Dryer FL. An updated comprehensive kinetic model of hydrogen combustion. Int J Chem Kinet 2004;36:566-75.

[40] Chase MW. NIST-JANAF thermochemical tables 2 volume-set (journal of physical and chemical reference data monograph 9). Maryland: American Institute of Physics; 1998.

[41] Kee RJ, Rupley FM, Miller JA. Chemkin-II: a fortran chemical kinetics package for the analysis of gas-phase chemical kinetics. Sandia: Sandia National Laboratories; 1989.

[42] Bird RB, Stewart WE, Lightfoot EN. Viscosity and the mechanisms of momentum transport. Transport phenomena. 2nd ed. New York: John Wiley \& Sons; 2002. p. 27.

[43] Won-Wook K, Suresh M. A new dynamic one-equation subgrid-scale model for large eddy simulations. In: 33rd aerospace sciences meeting and exhibit. Reno, NV: American Institute of Aeronautics and Astronautics; 1995.

[44] Kim S-E. Large eddy simulation using an unstrcutured mesh based finite-volume solver. In: 34th AIAA fluid dynamics conference and exhibit. Portland, Oregon; 2004.

[45] Kim W-W, Menon S, Kim W-W, Menon S. Application of the localized dynamic subgrid-scale model to turbulent wallbounded flows. 1997.

[46] Liu S, Meneveau C, Katz J. On the properties of similarity subgrid-scale models as deduced from measurements in a turbulent jet. J Fluid Mech 1994;275:83-119.

[47] Fureby C. LES for supersonic combustion. In: 18th AIAA/3AF international space planes and hypersonic systems and technologies conference; 2012. Tours, France.

[48] Fureby C, Tabor G, Weller HG, Gosman AD. A comparative study of subgrid scale models in homogeneous isotropic turbulence. Phys Fluids 1997;9:1416-29.

[49] Fureby C, Gosman AD, Tabor G, Weller HG, Sandham UoS N, Southampton, Wolfshtein M. Large eddy simulation of turbulent channel flows. Turbulent shear flows 1997;11:28-33.

[50] Li X, Yao W, Fan X. Large-eddy simulation of time evolution and instability of highly underexpanded sonic jets. AIAA J 2016;54:3191-211.
[51] Smagorinsky J. General circulation experiments with the primitive equations. I. the basic experiment. Mon Weather Rev 1963;91:99-164.

[52] Weller HG, Tabor G, Jasak H, Fureby C. A tensorial approach to CFD using object oriented techniques. Comput Phys 1997;12:620-31.

[53] Li Q, Wang Z. Dynamic mode decomposition of turbulent combustion process in DLR scramjet combustor. J Aero Eng 2017;30.

[54] Huang Z, Zhao M, Zhang H. Modelling n-heptane dilute spray flames in a model supersonic combustor fueled by hydrogen. Fuel 2020:264.

[55] Huang Z, Zhang H. Investigations of autoignition and propagation of supersonic ethylene flames stabilized by a cavity. Appl Energy 2020:265.

[56] Wu K, Li X, Yao W, Fan X. Three-dimensional numerical study of the acoustic properties of a highly underexpanded jet. In: 20th AIAA international space planes and hypersonic systems and technologies conference. Glasgow, Scotland; 2015.

[57] Li X, Wu K, Yao W, Fan X. A comparative study of highly underexpanded nitrogen and hydrogen jets using large eddy simulation. In: 20th AIAA international space planes and hypersonic systems and technologies conference. Glasgow, Scotland; 2015.

[58] Greenshields CJ, Weller HG, Gasparini L, Reese JM. Implementation of semi-discrete, non-staggered central schemes in a colocated, polyhedral, finite volume framework, for high-speed viscous flows. Int J Numer Methods Fluid 2009;38:139-61.

[59] Lee Y, Yao W, Fan X. A low-dissipation solver based on OpenFOAM designed for large eddy simulation in compressible flows. In: 21st AIAA international space planes and hypersonics technologies conference. Xiamen, China; 2017.

[60] Wu K, Zhang P, Yao W, Fan X. LES study of flame stabilization in DLR hydrogen supersonic combustor with strut injection. In: 21st AIAA international space planes and hypersonics technologies conference. Xiamen, China; 2017.

[61] Wu K, Zhang P, Yao W, Fan X. Computational realization of multiple flame stabilization modes in DLR strut-injection hydrogen supersonic combustor. Proc Combust Inst 2019;37:3685-92.

[62] Yao W, Yuan Y, Li X, Wang J, Wu K, Fan X. Comparative study of elliptic and round scramjet combustors fueled by RP-3. J Propul Power 2018;34:772-86.

[63] Wu K, Contino F, Yao W, Fan X. On the application of tabulated dynamic adaptive chemistry in ethylene-fueled supersonic combustion. Combust Flame 2018;197:265-75.

[64] Wu K, Yao W, Fan X. Development and fidelity evaluation of a skeletal ethylene mechanism under scramjet-relevant conditions. Energy Fuel 2017;31:14296-305.

[65] Lee Y, Yao W, Fan X. Low-dissipative hybrid compressible solver designed for large eddy simulation of supersonic turbulent flows. AIAA J 2018;56:3086-96.

[66] Kurganov A, Tadmor E. New high-resolution central schemes for nonlinear conservation laws and convection-diffusion equations. J Comput Phys 2000;160:241-82.

[67] Kennedy CA, Gruber A. Reduced aliasing formulations of the convective terms within the Navier-Stokes equations for a compressible fluid. J Comput Phys 2008;227:1676-700.

[68] Vuorinen V, Larmi M, Schlatter P, Fuchs L, Boersma BJ. A low-dissipative, scale-selective discretization scheme for the Navier-Stokes equations. Comput Fluid 2012;70:195-205.

[69] Baba-Ahmadi MH, Tabor G. Inlet conditions for LES using mapping and feedback control. Comput Fluid 2009;38:1299-311. 
[70] Crank J, Nicotson E. A practical method for numerical evaluation of solutions of partial differential equations of the heat-conduction type. Adv Comput Math 1996;6:207-26.

[71] Jin T, Luo K, Lu S, Fan J. Direct numerical simulation of a supersonic lifted hydrogen jet flame: a priori study on combustion models. Acta Astronaut 2015;109:52-64.

[72] Jin T, Luo K, Lu S, Fan J. DNS investigation on flame structure and scalar dissipation of a supersonic lifted hydrogen jet flame in heated coflow. Int J Hydrogen Energy 2013;38:9886-96.

[73] Lu S, Fan J, Luo K. High-fidelity resolution of the characteristic structures of a supersonic hydrogen jet flame with heated co-flow air. Int J Hydrogen Energy 2012;37:3528-39.

[74] Clarke DK, Hassan HA, Salas MD. Euler calculations for multielement airfoils using Cartesian grids. AIAA J 1986;24:353-8.

[75] Jin T, Luo K, Lu S, Fan J. Analysis of conditional statistics of a supersonic jet flame in heated coflow via direct numerical simulation. Acta Astronaut 2017;134:179-88.

[76] Lu S. Direct numerical simulation of the combustion in supersonic turbulent flow. Zhejiang University; 2011. 